\title{
Biodiversity of Indian Rotifers (Rotifera) with remarks on biogeography and richness in diverse ecosystems
}

\author{
B. K. SHARMA ${ }^{1} \&$ S. SHARMA ${ }^{2}$ \\ ${ }^{1}$ Bhushan Kumar Sharma, Department of Zoology, North-Eastern Hill University, Shillong - 793 022, Meghalaya, India \\ ${ }^{2}$ Sumita Sharma, Lady Veronica Road, Shillong - 793 003, Meghalaya, India \\ Corresponding author: profbksharma@gmail.com
}

\begin{abstract}
We assess biodiversity status of Rotifera known from India to-date based on our studies from various regions of this country and evaluation of other viable records, and highlight notable features of biogeography and richness. The Indian fauna reveals 434 valid species belonging to 68 genera and 25 families and thus indicates the most biodiverse Rotifera vis-àvis south and Southeast Asia, and records $\sim 25 \%$ and $\sim 41 \%$ species of global and regional biogeographic interest. It depicts the littoral-periphytic nature, broadly tropical character, the limited reports of cold-water species from the sub-Himalayan and Himalayan latitudes, paucity of the endemics and Bdelloids, and cryptic diversity awaits analyses. The richest diversity and distinct biogeographic identity of Rotifera of Northeast India (NEI) is attributed to location of this region in the 'Himalayan and Indo-Burmese' biodiversity hot-spots, 'Assam gateway' - the biogeographic corridor, and the 'Rotiferologist effect'. Regional disparity and spatial heterogeneity of biodiversity elsewhere from India are attributed to the limited sampling, inadequate collections from diverse ecosystems, unidentified species, and paucity of attention on smaller species. The biodiverse rotifer assemblages of the floodplain lakes including Deepor Beel and Loktak Lake, the two Ramsar sites and globally megadiverse ecosystems, are hypothesized to habitat diversity of these ecotones, while 'Rotifera paradox' depict speciose constellations per sample. The species-rich small floodplain and urban wetlands focus interest on rotifer diversity in small water bodies. We estimate more diverse Indian Rotifera following analyses of collections from underexplored and unexplored regions and ecosystems, and the bdelloid and sessile rotifers using integrative taxonomic approaches.
\end{abstract}

Key words. Biodiverse rotifers, diverse environs, regional disparity, richness, Rotifera paradox

\section{INTRODUCTION}

$\mathrm{R}$ otifera or 'wheel animalcules' colonize diverse aquatic and semi-aquatic environs with endless profusion of body forms well adapted to their living habits and habitats. These features along with the latitudinal variations $v s$. 'tropic' and 'temperate' centered origin of various taxa (Pejler 1977, Dumont 1983, Segers 1996, 2001, 2008) characterize these metazoans as useful models for assessing patterns in global and regional biodiversity as well as biogeography analyses. The studies on the Indian Rotifera were initiated in West Bengal (Anderson 1889) and indicated useful earlier faunal surveys of Murray (1906), Edmondson \& Hutchinson (1934), Nayar (1968) and Wulfert (1966). Sharma \& Michael (1980) provided an overview of taxonomic studies until 1980's, while the state-of-art reports (Sharma
1991, 1996, 1998a) and useful but unvalidated compilation by Dhanapathi (2000) traced the subsequent faunal diversity progress which lacked a definite focus on biodiversity and biogeography until the end of the $20^{\text {th }}$ century. The period from beginning of the $21^{\text {st }}$ century to-date indicated notable Rotifera biodiversity works from the states of Arunachal Pradesh (Sharma \& Sharma 2019a), Jammu \& Kashmir (Sharma \& Sharma 2018a), Mizoram (Sharma \& Sharma 2015a), Nagaland (Sharma et al. 2017), and Tamil Nadu (Sharma \& Sharma 2009). Meta-diversity updates on NEI Rotifera (Sharma \& Sharma 2005, 2014a, 2019b), and the reviews on the Indian species of Lecanidae (Sharma \& Sharma 2014b), Brachionidae (Sharma \& Sharma 2014c), Lepadellidae (Sharma \& Sharma 2015b) and Testudinella (Sharma \& Sharma 2018b) notably added to our understanding of the rotifer diversity of India. This 
period also indicated certain important biodiversity studies from the floodplains of Assam (Sharma 2005, 2014; Sharma \& Sharma 2008, 2014d, 2019c, 2019d, Sharma et al. 2017, 2018) and Manipur (Sharma 2009a) states of NEI, and the river Yamuna floodplains (Arora \& Mehra 2003). Besides, our studies provided useful database for meta-analysis of Rotifera diversity of Deepor beel (Sharma \& Sharma 2011, 2012, 2013, 2015c) and Loktak Lake (Sharma 2009b; Sharma \& Sharma 2018c; Sharma et al. 2016) - the two intensively sampled Ramsar sites of India.

This study aims to present a critical appraisal of the current biodiversity status of the Indian Rotifera based on our contributions from various regions of India vis-a-vis intensive sampling from $\mathrm{NEI}$; the semi-intensive collections from Jammu \& Kashmir (western Himalayas) and West Bengal (east India) and Tamil Nadu (south India); the studies (BKS, unpublished) from central, east and south India; and evaluation of other viable reports published over the last nearly 130 years. A detailed systematic list of 434 valid Rotifera species known to-date from India is presented. Comments are made on biodiversity and biogeography of the Indian Rotifera with reference to nature and composition, species of global and regional distribution significance, spatial heterogeneity and regional disparity of the biodiversity, and species richness of the rotifer assemblages in various aquatic ecosystems. In addition, we highlight existing lacunae and suggest areas needing attention for the future biodiversity studies on the taxon.

\section{MATERIALS AND METHODS}

This biodiversity assessment is primarily based on analyses of our plankton and semi-planktonic samples collected from the diverse lentic environs of various regions of India over the last three decades. In addition, we undertook validation of the viable taxa vide the published reports. All our collections were obtained by towing nylobolt

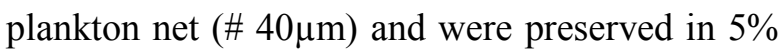
formalin. Voucher collections are deposited in the national holdings of Zoological Survey of India, Kolkata. We examined the collections from the following regions / states of India (Fig. 1):

1. Northeast India - Arunachal Pradesh, Assam, Manipur, Mizoram, Meghalaya, Nagaland and Tripura (Fig. 2).

2. Eastern India - West Bengal, Odisha (Orissa), Bihar and Jharkhand.

3. North India - Jammu \& Kashmir, Panjab, Haryana.

4. Central India - Madhya Pradesh, Maharashtra and Goa.

5. South India - Andhra Pradesh, Telangana, Tamil Nadu, Karnataka and Kerala.

6. Insular freshwaters off the south Andamans Islands.

Microphotographs were taken by a Leica (DM 1000) stereoscopic phase contrast microscope fitted with an image analyzer and the figures were drawn with a drawing tube attachment. Rotifera species were identified following Koste (1978), Koste \& Shiel $(1987,1989,1990)$, Shiel \& Koste (1992), Segers (1995, 1996), Sharma (1983, 1987a, 1987b, 1998b), Sharma \& Sharma (1987, 1999, 2000, 2008, 2013, 2014b, 2014c, 2015a, 2019b) and Jersabek and Leitner (2013). Segers (2002, 2007) and Jersabek \& Leitner (2013) were followed for classification, nomenclature, and biogeography of the taxon. Unless indicated otherwise, the figures and the micro-photographs are based on our collections from different parts of India.

\section{RESULTS}

A total of 434 Rotifera species belonging to 25 families and 68 genera are considered as validly known from India based on our present biodiversity assessment. A detailed systematic list of the documented taxa is presented hereunder: 


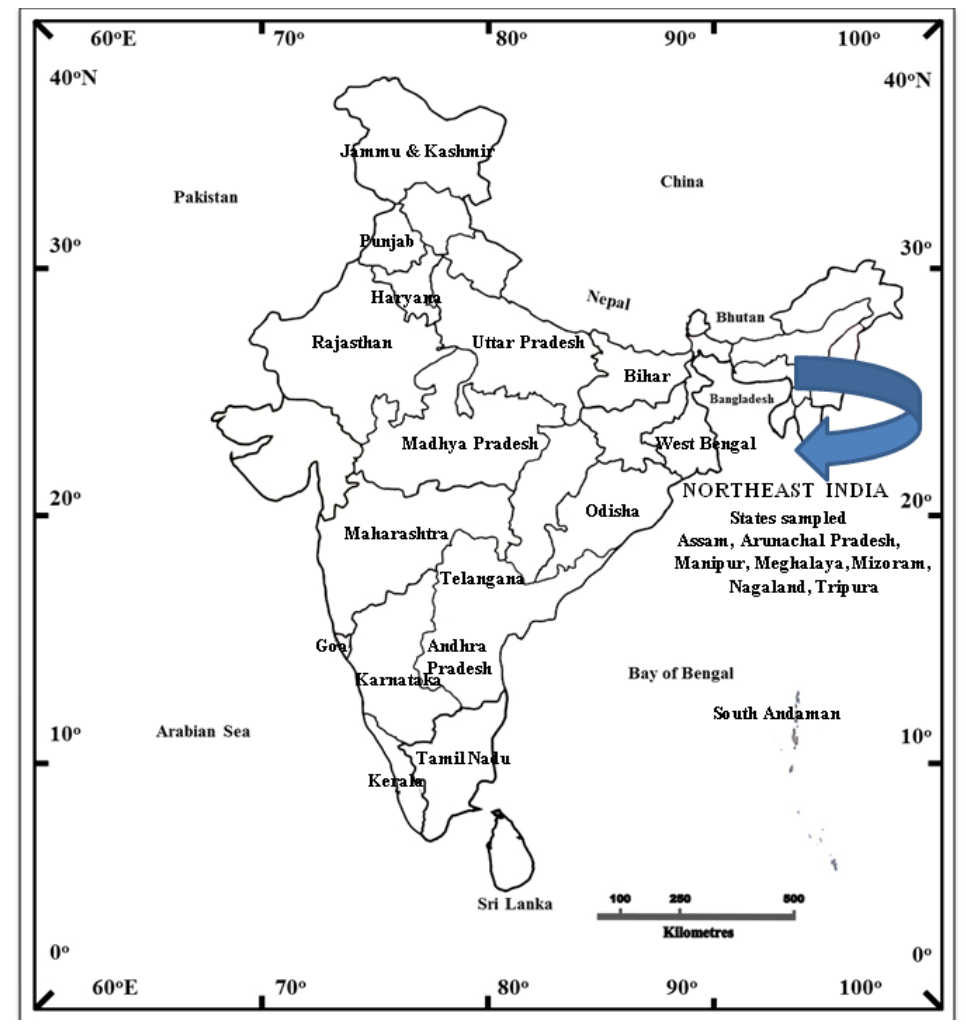

Figure 1. Map of India showing the sampled states

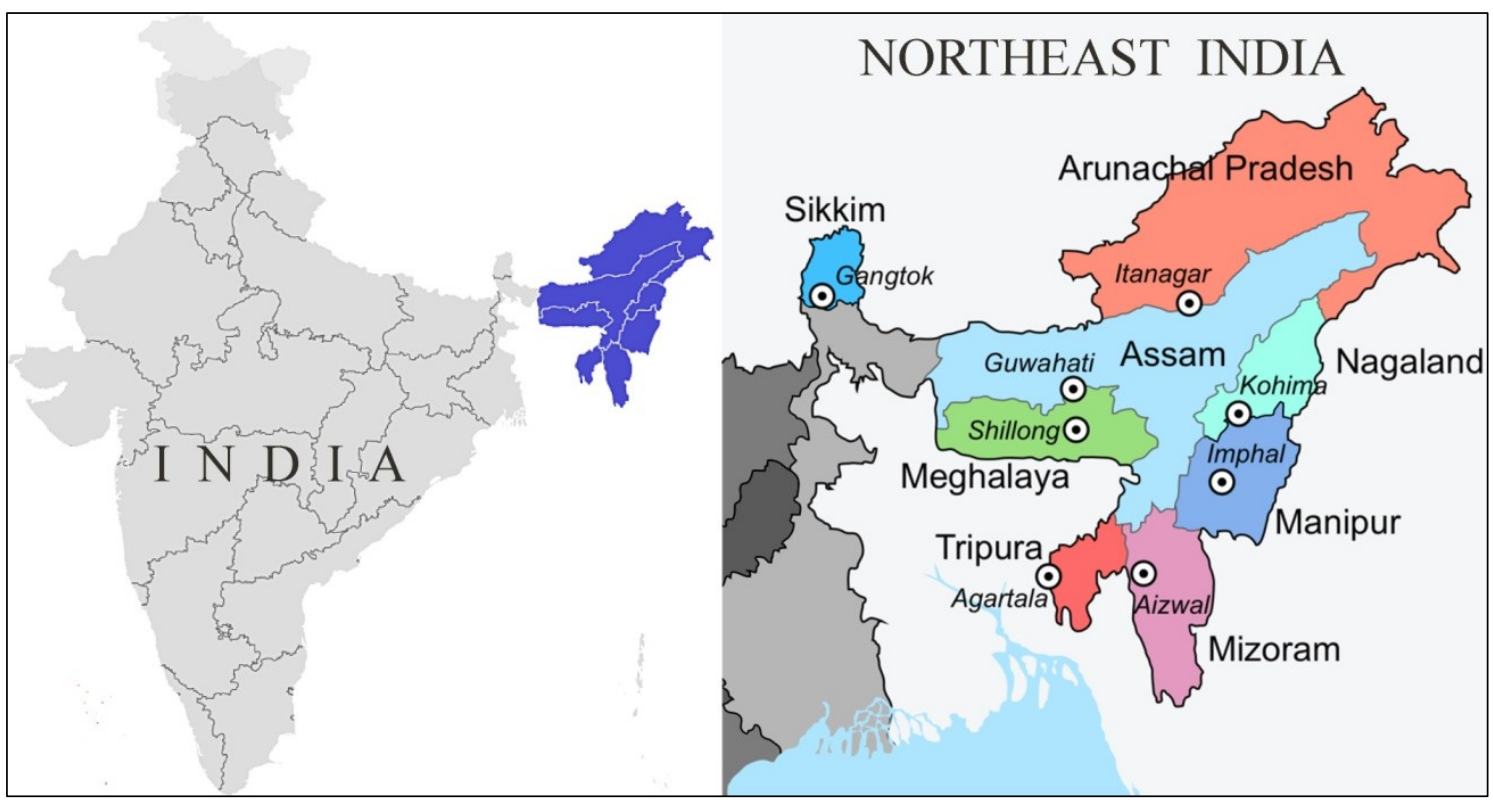

Figure 2. A = Map of India showing northeast India (blue color), B = map of NEI indicating the sampled states of Arunachal Pradesh, Assam, Manipur, Meghalaya, Mizoram, Nagaland and Tripura (After Sharma \& Sharma 2019b) 


\section{Systematic list of Rotifera recorded from India}

\section{Phylum: Rotifera \\ Class: Eurotatoria \\ Subclass: Monogononta}

\section{Order: Ploima}

Family: Brachionidae

1. Anuraeopsis coelata De Beauchamp, 1932 *

2. A. fissa (Gosse, 1851)*

3. A. navicula Rousselet, 1911*

4. Brachionus ahlstromi Lindeman, 1939*

5. B. angularis Gosse, 1851 s. lato *

B. angularis bidens Plate, 1886 *

6. B. bennini Leissling, 1924 *

7. B. bidentatus Anderson, 1889 s. lato *

8. B. budapestinensis Daday, 1885 *

9. B. calyciflorus Pallas, 1766 s. lato *

10. B. caudatus Barrois \& Daday, 1894 s. lato*

11. B. dichotomus reductus Koste \& Shiel, 1980 *

12. B. dimidiatus Bryce, 1931 *

13. B. diversicornis (Daday, 1883) s. lato*

14. B. donneri Brehm, 1951 *

15. B. dorcas Gosse, $1851^{*}$

16. B. durgae Dhanapathi, 1974 *

17. B. falcatus Zacharias, 1898 s. lato* B. falcatus reductus Koste \& Shiel, 1987 *

18. B. forficula Wierzejski, 1891 s. lato *

19. B. kostei Shiel, 1983 *

20. B. leydigii Cohn, 1862

21. B. lyratus Shephard, $1911^{*}$

22. B. mirabilis Daday, 1897 *

23. B. murphyi Sudzuki, 1989 *

24. B. nilsoni Ahlstrom, 1940 *

25. B. plicatilis O.F. Muller, 1786 s. lato*

26. B. pterodinoides Rousselet, 1913 *

27. B. quadridentatus Hermann, 1783 s. lato*

28. B. rotundiformis Tschugunoff, $1921 *$

29. B. rubens Ehrenberg, 1838 *

30. B. sessilis Varga, 1951

31. B. srisumonae Segers, Kotethip \& Sanoamuang, 2004 *

32. B. urceolaris O.F. Muller, $1773 *$

33. Kellicottia longispina (Kellicott, 1879) *

34. Keratella cochlearis (Gosse, 1851) s. lato *

35. K. edmondsoni Ahlstrom, 1943

36. K. javana Hauer, 1937 *

37. K. hiemalis Carlin, $1943 *$

38. K. lenzi Hauer, 1953 *

39. K. procurva (Thorpe, 1891)*

40. K. quadrata (O.F. Muller, 1786) *

41. K. serrulata (Ehrenberg, 1838)*

42. K. tecta (Gosse, 1851)
43. K. ticinensis (Callerio, 1921)*

44. K. tropica (Apstein, 1907) s. lato *

45. Notholca acuminata (Ehrenberg, 1832)*

46. N. labis Gosse, 1887

47. N. squamula (O.F. Muller, 1786)*

48. N. striata (Müller, 1786)*

49. Plationus patulus (O.F. Muller, 1786) s. lato*

50. Platyias leloupi (Gillard, 1967)

51. P. quadricornis (Ehrenberg, 1832) * P. quadricornis andhraensis Dhanapathi, 1974 *

\section{Family: Epiphanidae}

52. Cyrtonia tuba (Ehrenberg, 1834)

53. Epiphanes brachionus (Ehrenberg, 1837) s. lato * E. brachionus spinosa (Rousselet, 1901) *

54. E. clavatula (Ehrenberg, 1831)*

55. E. macroura (Barrois \& Daday, 1894)*

56. E. senta (O.F. Muller, 1773)*

57. Mikrocodides chlaena (Gosse, 1886)

58. Proalides subtilis Rodewald, 1940

\section{Family: Euchlanidae}

59. Beauchampiella eudactylota (Gosse, 1886) *

60. Dipleuchlanis ornata Segers, 1993 *

61. D. propatula (Gosse, 1886) *

62. Euchlanis alata Voronkov, 1912

63. E. deflexa Gosse, 1851

64. E. dilatata Ehrenberg, 1832 s. lato *

65. E. incisa Carlin, 1939 *

66. E. meneta Myers, 1930 *

67. E. oropha Gosse, 1887 *

68. E. semicarinata Segers, 1993 *

69. E. triquetra Ehrenberg, 1838 *

70. Pseudoeuchlanis longipedes Dhanapathi, 1978 *

71. Tripleuchlanis plicata (Levander, 1894)*

\section{Family: Mytilinidae}

72. Lophocharis naias Wulfert, 1942

73. L. oxysternon (Gosse, 1851) *

74. L. salpina (Ehrenberg, 1834) *

75. Mytilina acanthophora Hauer, 1938 *

76. M. bisulcata (Lucks, 1912) *

77. M. brevispina (Ehrenberg, 1830)*

78. M. lobata Pourriot, 1996 *

79. M. michelangellii Reid \& Turner, $1988 *$

80. M. mucronata (O.F. Muller, 1773) *

81. M. ventralis (Ehrenberg, 1830) s. lato *

\section{Family: Trichotriidae}

82. Macrochaetus collinsi (Gosse, 1867) *

83. M. danneelae Koste \& Shiel, 1983 *

84. M. longipes Myers, 1934 *

85. M. sericus (Thorpe, 1893) * 
86. M. subquadratus Perty, 1850 *

87. Trichotria pocillum (O.F. Muller, 1776) *

88. T. tetractis (Ehrenberg, 1830) s. lato *

89. Wolga spinifera (Western, 1894)*

\section{Family: Lepadellidae}

90. Colurella adriatica Ehrenberg, 1831*

91. C. colurus (Ehrenberg, 1830) s. lato*

92. C. obtusa (Gosse, 1886) s. lato *

93. C. oxycauda Carlin, 1939

94. C. sanoamuangae Chittapun, Pholpunthin \& Segers, $1999^{*}$

95. C. sulcata (Stenroos, 1898)*

96. C. tesselata (Glascott, 1893)*

97. C. uncinata (O.F. Muller, 1773) s. lato *

98. Lepadella acuminata (Ehrenberg, 1834) s. lato*

99. L. apsicora Myers, 1934 *

100. L. apsida Harring, 1916 *

101. L benjamini Harring, 1916 *

102. L. bicornis Vasisht \& Battish, 1971 *

103. L. biloba Hauer, 1958 *

104. L. costatoides Segers, 1992 s. lato*

105. L. cristata (Rousselet, 1893) *

106. L. dactyliseta (Stenroos, 1898) *

107. L. desmeti Segers \& Chittapun, 2001*

108. L. discoidea Segers, 1993 *

109. L. ehrenbergi (Perty, 1850) *

110. L. elongata Koste, 1992 *

111. L. eurysterna Myers, 1942 *

112. L. cf. favorita Klement, $1962 *$

113. L. heterodactyla Fadeew, $1925 *$

114. L. heterostyla (Murray, 1913) *

115. L. imbricata Harring, 1914 *

116. L. kostei Wulfert, 1966

117. L. latusinus (Hilgendorf, 1889) *

118. L. lindaui Koste, $1981^{*}$

119. L. longiseta Myers, 1934*

120. L. minoruoides Koste \& Robertson, 1983 *

121. L. minuta (Weber \& Montet, 1918) *

122. L. nartiangensis Sharma \& Sharma, 1987 *

123. L. neglecta Segers \& Dumont, 1995 *

124. L. ovalis (O.F. Muller, 1786) s. lato*

125. L. patella patella (O.F. Muller, 1773) s. lato *

L. patella oblonga (Ehrenberg, 1834) *

L. patella persimilis De Ridder, 1961

126. L. quadricarinata (Stenroos, 1898) *

127. L. quinquecostata (Lucks, 1912) *

128. L. rhomboides (Gosse, 1886) s. lato *

129. L. rhomboidula (Bryce, 1890) *

130. L. triba Myers, 1934 *

131. L. triptera Ehrenberg, 1832 s. lato*

132. L. vandenbrandei Gillard, 1952 *

133. Squatinella bifurca (Bolton, 1884) *

134. S. lamellaris (O. F. Müller, 1786) *

\section{Family: Lecanidae}

135. Lecane acanthinula (Hauer, 1938) *

136. L. aculeata (Jakubski, 1912) *

137. L. arcuata (Bryce, 1891)*

138. L. arcula Harring, 1914 *

139. L. aeganea Harring, 1914 *

140. L. aspasia Myers, 1917 *

141. L. batillifer (Murray, 1913)*

142. L. bifastigata Hauer, 1938 *

143. L. bifurca (Bryce, 1892) *

144. L. blachei Berzins, 1973 *

145. L. braumi Koste, 1988 *

146. L. bulla bulla (Gosse, 1851) s. lato * L. bulla diabolica (Hauer, 1936) *

147. L. calcaria Harring \& Myers, $1926^{*}$

148. L. clara (Bryce, 1892) *

149. L. closterocerca (Schmarda, 1859) s. lato *

150. L. cornuta (Müller, 1786) s. lato *

151. L. crenata (Harring, 1913) *

152. L. crepida Harring, 1914 *

153. L. curvicornis (Murray, 1913) s. lato*

154. L. decipiens (Murray, 1913)*

155. L. depressa (Bryce, 1891)

156. L. dorysimilis Trinh Dang, Segers \& Sanoamuang, 2015*

157. L. doryssa Harring, 1914 *

158. L. elasma Harring \& Myers, 1926

159. L. elegans Harring, 1914 *

160. L. elongata Harring \& Myers, 1926 *

161. L. eswari Dhanapathi, 1976 *

162. L. flexilis (Gosse, 1886) s. lato*

163. L. furcata (Murray, 1913) s. lato*

164. L. galeata (Bryce, 1892)

165. L. glypta Harring \& Myers, 1926 *

166. L. haliclysta Harring \& Myers, 1926 *

167. L. hamata (Stokes, 1896) s. lato*

168. L. hastata (Murray, 1913) *

169. L. hornemanni (Ehrenberg, 1834) *

170. L. inermis (Bryce, 1892) *

171. L. inopinata Harring \& Myers, 1926 *

172. L. isanensis Sanoamuang \& Savatenalinton, $2001 *$

173. L. jaintiaensis Sharma, 1987 *

174. L. lateralis Sharma, 1978 *

175. L. latissima Yamamoto, 1951 *

176. L. leontina (Turner, 1892) s. lato*

177. L. levistyla (Olofsson, 1917)*

178. L. ligona (Dunlop, 1901) *

179. L. ludwigii (Eckstein, 1883) s. lato *

180. L. luna (Müller, 1776) s. lato *

181. L. lunaris (Ehrenberg, 1832) s. lato*

182. L. marchantaria Koste \& Robertson, 1983 *

183. L. monostyla (Daday, 1897) s. lato *

184. L. nana (Murray, 1913)*

185. L. nitida (Murray, 1913) *

186. L. niwati Segers, Kothetip \& Sanoamuang, 2004* 
187. L. obtusa (Murray, 1913) s. lato *

188. L. ohioensis (Herrick, 1885) *

189. L. papuana (Murray, 1913) s. lato*

190. L. pawlowskii Wulfert, 1966 *

191. L. paxiana Hauer, 1940 *

192. L. perplexa (Ahlstrom, 1938)

193. L. pertica Harring \& Myers, 1926 *

194. L. ploenensis (Voigt, 1902)*

195. L. punctata (Murray, 1913)

196. L. pusilla Harring, 1914 *

197. L. pyriformis (Daday, 1905) *

198. L. quadridentata (Ehrenberg,1830) s. lato *

199. L. rhenana Hauer, 1929 *

200. L. rhytida Harring \& Myers, 1926 *

201. L. rugosa (Harring, 1914) *

202. L. ruttneri Hauer, 1938 *

203. L. schraederi Wulfert, 1966 *

204. L. scutata (Harring \& Myers, 1926) *

205. L. shieli Segers \& Sanoamuang, 1994 *

206. L. signifera (Jennings, 1896) s. lato *

207. L. simonneae Segers, 1993 *

208. L. sinuata (Hauer, 1938) *

209. L. sola Hauer, 1936 *

210. L. solfatara (Hauer, 1938) *

211. L. stichaea Harring, 1913 *

212. L. stichoclysta Segers, 1993 *

213. L. stenroosi (Meissner, 1908) *

214. L. stokesii (Pell, 1890) *

215. L. styrax (Harring \& Myers, 1926) *

216. L. superaculeata Sanoamuang \& Segers, $1997 *$

217. L. sympoda Hauer, 1929 *

218. L. syngenes (Hauer, 1938) *

219. L. tenuiseta Harring, 1914 *

220. L. thalera (Harring \& Myers, 1926)

221. L. thienemanni (Hauer, 1938) *

222. L. tryphema Harring \& Myers, 1926

223. L. undulata Hauer, 1938 *

224. L unguitata (Fadeev, 1925) s. lato *

225. L. ungulata (Gosse, 1887) s. lato*

226. L. vasishti Sharma, 1980 *

227. L. verecunda Harring \& Myers, 1926 *

Family: Proalidae

228. Proales decipiens (Ehrenberg, 1832)

229. P. fallaciosa Wulfert, 1937

230. P. indirae Wulfert, 1966

\section{Family: Notommatidae}

231. Cephalodella auriculata (O.F. Müller, 1773) *

232. C. catellina (O.F. Müller, 1786) *

233. C. exigua (Gosse, 1886)*

234. C. forficata (Ehrenberg, 1832) *

235. C. forficula (Ehrenberg, 1838) *

236. C. gibba (Ehrenberg, 1830)*
237. C. gigantea Remane, 1933

238. C. hiulca Myers, 1924

239. C. intuta Myers, $1924^{*}$

240. C. megalocephala (Glascott, 1893)

241. C. misgurnus Wulfert, 1937

242. C. mucronata Myers, 1924 *

243. C. panarista Myers, $1924 *$

244. C. trigona (Rousselet, 1895)*

245. C. ventripes (Dixon-Nuttall, 1901) *

246. Eosphora anthadis Harring \& Myers, 1922

247. E. najas Ehrenberg, 1830

248. Itura aurita (Ehrenberg, 1830)

249. Monommata actices Myers, 1930

250. M. grandis Tessin, 1890 *

251. M. longiseta (O.F. Müller, 1786) *

252. M. maculata Harring \& Myers, 1930 *

253. Notommata aurita (Müller, 1786)

254. N.copeus Ehrenberg, 1834 *

255. N. glyphura Wulfert, 1935 *

256. N. pachyura (Gosse, 1886) *

257. N. pseudocerberus De Beauchamp, 1908

258. N. saccigera Ehrenberg, 1830 *

259. N. spinata Koste \& Shiel, 1991*

260. N. tripus Ehrenberg, 1838 *

261. Taphrocampa annulosa Gosse, 1851 *

262. T. selenura Gosse, 1887 *

\section{Family: Scaridiidae}

263. Scaridium longicaudum (O.F. Müller, 1786) *

\section{Family: Gastropodidae}

264. Ascomorpha. ecaudis Perty, 1850 *

265. A. saltans Bartsch, 1870 *

A. saltans indica Wulfert, 1966

266. A. ovalis (Bergendal, 1892)*

267. Gastropus hyptopus (Ehrenberg, 1838) *

268. G. minor (Rousselet, 1892) *

269. G. stylifer Imhof, 1891*

\section{Family: Trichocercidae}

270. Ascomorphella volvocicola (Plate, 1886)

271. Trichocerca abilioi Segers \& Sarma, 1993 *

272. T. bicristata (Gosse, 1887) *

273. T. bidens (Lucks, 1912) *

274. T. brachyura (Gosse, 1851)

275. T. braziliensis (Murray, 1913)*

276. T. capucina (Wierzejski \& Zacharias, 1893) *

277. T. cavia (Gosse, 1886)

278. T. chattoni (De Beauchamp, 1907)

279. T. cylindrica (Imhof, 1891) *

280. T. edmondsoni (Myers, 1936) *

281. T. elongata (Gosse, 1886) *

282. T. flagellata Hauer, 1937 *

283. T. hollaerti De Smet, 1990 * 
284. T. iernis (Gosse, 1887) *

285. $T$ insignis (Herrick, 1885)*

286. T. insulana (Hauer, 1937) *

287. T. kostei Segers, $1993 *$

288. T. longiseta (Schrank, $1802 *$

289. T. maior (Hauer, 1935)*

290. T. mus Hauer, 1938 *

291. T. myersi (Hauer, 1931)

292. T. porcellus (Gosse, 1881)*

293. T. pusilla (Jennings, 1903)*

294. T. rattus (O.F. Müller, 1776) s. lato *

295. T. ruttneri Donner, 1953 *

296. T. scipio (Gosse, 1886) *

297. T. siamensis Segers \& Pholpunthin, 1997 *

298. T. similis (Wierzejski, 1893) s. lato *

299. T. stylata (Gosse, 1851)*

300. T. sulcata (Jennings, 1894) *

301. T. taurocephala (Hauer, 1931)

302. T. tenuior (Gosse, 1886) *

303. T. tigris (O.F. Müller, 1786)

304. T. uncinata (Voigt, 1902) *

305. T. voluta (Murray, 1913) *

306. T. weberi (Jennings, 1903) *

\section{Family: Asplanchnidae}

307. Asplanchna brightwelli Gosse, 1850 *

308. A. herricki de Guerne, 1888

309. A. intermedia Hudson, 1886 *

310. A. priodonta Gosse, 1850 *

311. A. sieboldii (Leydig, 1854)*

312. Asplanchnopus bhimavaramensis Dhanapathi, 1975 *

313. A. hyalinus Harring, 1913 *

314. A. multiceps (Schrank, 1793)

\section{Family: Synchaetidae}

315. Ploesoma hudsoni (Imhof, 1891) *

316. P. lenticulare Herrick, 1885 *

317. Polyarthra euryptera Wierzejski, 1891*

318. P. cf. dolichoptera Idelson, 1925 *

319. P. indica Segers \& Babu, 1999

320. P. vulgaris Carlin, 1943 s. lato*

321. Synchaeta oblonga Ehrenberg, 1832 *

322. S. longipes Gosse, 1887 *

323. S. pectinata Ehrenberg, 1832 *

324. S. stylata Wierzejski, 1893 *

325. S. tremula (O.F. Müller, 1786)

\section{Family: Dicranophoridae}

326. Dicranophoroides caudatus (Ehrenberg, 1834) *

327. Dicranophorus dolerus Harring \& Myers, 1928 *

328. D. epicharis Harring \& Myers, 1928 *

329. D. forcipatus (O.F. Müller, 1786) *

330. D. luetkeni (Bergendal, 1892)

331. D. myriophylli (Harring, 1913)
332. D. tegillus Harring \& Myers, 1928

333. Encentrum longipes Wulfert, 1936

Order: Flosculariaceae

Family: Floscularidae

334. Beauchampia crucigera (Dutrochet, 1812)

335. Floscularia conifera (Hudson, 1886) *

336. F. ringens (Linnaeus, 1758) s. lato *

337. Lacinularia flosculosa (O.F. Müller, 1773) *

338. L. racemovata Thorpe, 1893 *

339. Limnias ceratophylli Schrank, 1803 s. lato *

340. L. melicerta Weisse, 1848

341. Ptygura barbata (Edmondson, 1939)

342. P. furcillata (Kellicott, 1889)

343. P. kostei José De Paggi, 1996

344. P. melicerta Ehrenberg, 1832 *

345. P. pedunculata Edmondson, 1939

346. P. stephanion (Anderson, 1889)

347. P. tacita Edmondson, 1940 *

348. Sinantherina procera (Thorpe, 1893)

349. S. semibullata (Thorpe, 1893)*

350. S. socialis (Linne, 1758) *

351. S. spinosa (Thorpe, 1893)*

352. Stephanoceros fimbriatus (Goldfusz, 1820) *

\section{Family: Conochilidae}

353. Conochilus dossuarius Hudson, 1885 *

354. C. natans Seligo 1901*

355. C. hippocrepis (Schrank, 1803)

356. C. unicornis Rousselet, 1892*

\section{Family: Hexarthridae}

357. Hexarthra bulgarica (Wiszniewski, 1933)

358. H. intermedia (Wiszniewski, 1929) *

359. H. mira (Hudson, 1871) *

360. H. oxyuris (Zernov, 1903)

Family: Testudinellidae

361. Pompholyx complanata Gosse, $1851^{*}$

362. P. sulcata Hudson, 1885 *

363. Testudinella amphora Hauer, 1938 s. lato *

364. T. brevicaudata Yamamoto, 1951 *

365. T. dendradena de Beauchamp, 1955 *

366. T. emarginula (Stenroos, 1898) s. lato *

367. T. greeni Koste, $1981 *$

368. T. incisa (Ternetz, 1892)

369. T. insinuata Hauer, 1938 *

370. T. mucronata (Gosse, 1886) *

371. T. parva parva (Ternetz, 1892)

T. parva bidentata (Ternetz, 1892) *

T. parva semiparva Hauer, 1938 *

372. T. patina (Hermann, 1783) s. lato*

373. T. tridentata Smirnov, 1931 s. lato*

374. T. walkeri Koste \& Shiel, 1980 * 
375. T. sp. Sharma \& Sharma $2018 \mathrm{~b} *$

376. T. sp.1 Sharma \& Sharma $2018 \mathrm{~b}$ *

\section{Family: Trochosphaeridae}

377. Filinia brachiata (Rousselet, 1901) *

378. F. camasecla Myers, 1938 *

379. F. cornuta (Weisse, 1848) *

380. F. longiseta (Ehrenberg, 1834) s. lato *

381. F. opoliensis (Zacharias, 1898) *

382. F. pejleri Hutchinson, 1964 *

383. F. saltator (Gosse, 1886) *

384. F. terminalis (Plate, 1886) s. lato *

385. Horaella brehmi Donner, 1949 *

386. Trochosphaera aequatorialis Semper, 1872 *

387. T. solstitialis Thorpe, 1893 *

\section{Order: Collothecaceae}

Family: Atrochidae

388. Cupelopagis vorax (Leidy, 1857)*

\section{Family: Collothecidae}

389. Collotheca ambigua (Hudson, 1883)

390. C. campanulata (Dobie, 1849)

391. C. hexalobata Banik, 2000

392. C. mutabilis (Hudson, 1885)

393. C. ornata (Ehrenberg, 1832)*

394. C. tetralobata Banik, 2000

395. C. tenuilobata (Anderson, 1889)

396. C. trilobata (Collins, 1872)

\section{Subclass: Bdelloidea}

Family: Adinetidae

397. Adineta vaga major Bryce, 1893 *

A. vaga minor Bryce, 1893

398. A. longicornis Murray, 1906

\section{Family: Habrotrochidae}

399. Habrotrocha angusticollis (Murray, 1905) * H. angusticollis attenuata (Murray, 1906) *

Of the listed Indian Rotifera, 359 species belonging to 25 families and 67 genera (Table 1) are observed in our plankton and semi-plankton collections from the different regions / states of India (Fig. 3), while 303 species (Fig. 4) belonging to 53 genera and 24 families are observed from seven states of NEI. The monogonont rotifers include 396 belonging to 22 families and 61 genera, and 339 belonging to 21 families and 53 genera from India and in our collections, respectively (Table 1). The bdelloid rotifers
400. H. aspera (Bryce, 1892)

401. H. bidens (Gosse, 1851)

402. H. lata (Bryce, 1892)

403. H. leitgebii (Zelinka, 1886)

404. H. microcephala (Murray, 1906)

405. H. nodosa (Murray, 1906)

406. H. perforata (Murray, 1906)

\section{Family: Philodinidae}

407. Dissotrocha aculeata (Ehrenberg, 1832) *

408. Embata laticeps (Murray, 1905)

409. Macrotrachela bullata (Murray, 1906)

410. M. formosa (Murray, 1906)

411. M. habita (Bryce, 1894)

412. M. multispinosa Thompson, 1892 *

413. M. musculosa (Milne, 1886)

414. M. papillosa Thompson, 1892

415. M. plicata (Bryce, 1892)

416. M. quadricornifera rigida Milne, 1916

417. Philodina brevipes Murray, 1902

418. P. citrina Ehrenberg, 1832*

419. P. flaviceps Bryce, 1906

420. P. indica Murray, 1906

421. P. megalotrocha Ehrenberg, 1832

422. P. roseola Ehrenberg, 1832

423. P. squamosa Murray, 1906

424. P. vorax (Janson, 1893)

425. Rotaria citrina (Ehrenberg, 1838) *

426. R. macroceros (Gosse, 1851)

427. R. mento (Anderson, 1889) *

428. R. neptunia (Ehrenberg, 1830) *

429. R. neptunoida Harring, 1913*

430. R. ovata (Anderson, 1889)

431. R. rotatoria (Pallas, 1766) *

432. R. sordida sordida (Western, 1893)

R. sordida fimbriata (Murray, 1906)

433. R. tardigrada (Ehrenberg, 1830)

434. R. tridens (Montet, 1915)

*Recorded in our collections from different regions of India

(Table 1) are represented by 38 species belonging to 3 families and 7 genera, while our samples reveal 11 species and four genera of bdelloids. Rotifera of NEI reveals 287 species of Monogononta and 16 species of Bdelloidea.

Lecanidae $(93$ species $)>$ Brachionidae $(51$ species $)>$ Lepadellidae $(45$ species $)>$ Trichocercidae (37 species) $>$ Notommatidae (32 species) and Philodinidae (27) are notable, families, while Floscularidae (19 species), Testudinellidae 
(16 species), Euchlanidae (13 species), Synchaetidae (11 species), Trochosphaeridae (11 species) and Mytilinidae (10 species) are noteworthy. Lecane (93 species) is most speciose genus; Trichocerca (36 species) $\geq$ Lepadella (35 species) $>$ Brachionus (30 species) are notable, while Cephalodella (15 species) $\geq$ Testudinella (14 species) $>$ Keratella $(11$ species $) \geq$ Rotaria (10 species) are other important genera; Euchlanis, Colurella, Notommata, Filinia, Collotheca, Habrotrocha, Macrotrachela and Philodina include eight species each and Mytilina includes seven species.
The family-wise breakup of the rotifer taxa known from India and in our collections are indicated in Table 1. The species observed in our collections from the different states of India are indicated in Fig. 3. Our intensive samples from seven states of NEI (Fig. 4) indicate the rotifer richness ranging between $181 \pm 39$ species with 244, 200, 176, 172, 162, 161 and 150 species observed from Assam, Manipur, Tripura, Arunachal Pradesh, Mizoram, Meghalaya and Nagaland, respectively.

Table 1: Family-wise composition of Rotifera

\begin{tabular}{|c|c|c|c|c|}
\hline \multirow{2}{*}{ Families $\downarrow \quad$ Taxa $\rightarrow$} & \multicolumn{2}{|c|}{ Species } & \multicolumn{2}{|c|}{ Genera } \\
\hline & India & $\begin{array}{c}\text { Our } \\
\text { collections }\end{array}$ & India & $\begin{array}{c}\text { Our } \\
\text { collections }\end{array}$ \\
\hline \multicolumn{5}{|c|}{ Subclass : Monogononta } \\
\hline \multicolumn{5}{|c|}{ Order : Ploima } \\
\hline Family: Brachionidae & 51 & 50 & 7 & 7 \\
\hline Family: Epiphanidae & 07 & 04 & 4 & 1 \\
\hline Family: Euchlanidae & 13 & 11 & 5 & 5 \\
\hline Family: Mytilinidae & 10 & 09 & 2 & 2 \\
\hline Family: Trichotriidae & 08 & 08 & 3 & 3 \\
\hline Family: Lepadellidae & 45 & 43 & 3 & 3 \\
\hline Family: Lecanidae & 93 & 88 & 1 & 1 \\
\hline Family: Proalidae & 03 & 0 & 1 & 0 \\
\hline Family: Notommatidae & 32 & 22 & 6 & 4 \\
\hline Family: Scaridiidae & 01 & 01 & 1 & 1 \\
\hline Family: Gastropodidae & 06 & 06 & 2 & 2 \\
\hline Family: Trichocercidae & 37 & 32 & 2 & 2 \\
\hline Family: Asplanchnidae & 08 & 06 & 2 & 2 \\
\hline Family: Synchaetidae & 11 & 09 & 3 & 3 \\
\hline Family: Dicranophoridae & 08 & 05 & 3 & 2 \\
\hline \multicolumn{5}{|c|}{ Order: Flosculariaceae } \\
\hline Family: Floscularidae & 19 & 11 & 7 & 6 \\
\hline Family: Conochilidae & 04 & 03 & 1 & 1 \\
\hline Family: Hexarthridae & 04 & 02 & 1 & 1 \\
\hline Family: Testudinellidae & 16 & 15 & 2 & 2 \\
\hline Family: Trochosphaeridae & 11 & 11 & 3 & 3 \\
\hline \multicolumn{5}{|c|}{ Order: Collothecaceae } \\
\hline Family: Atrochidae & 01 & 01 & 1 & 1 \\
\hline Family: Collothecidae & 08 & 02 & 1 & 1 \\
\hline \multicolumn{5}{|c|}{ Sub-class: Bdelloidea } \\
\hline Family: Adinetidae & 02 & 01 & 1 & 1 \\
\hline Family: Habrotrochidae & 08 & 01 & 1 & 1 \\
\hline Family: Philodinidae & 28 & 09 & 5 & 4 \\
\hline Total Rotifer taxa & 434 & 350 & 68 & 59 \\
\hline
\end{tabular}




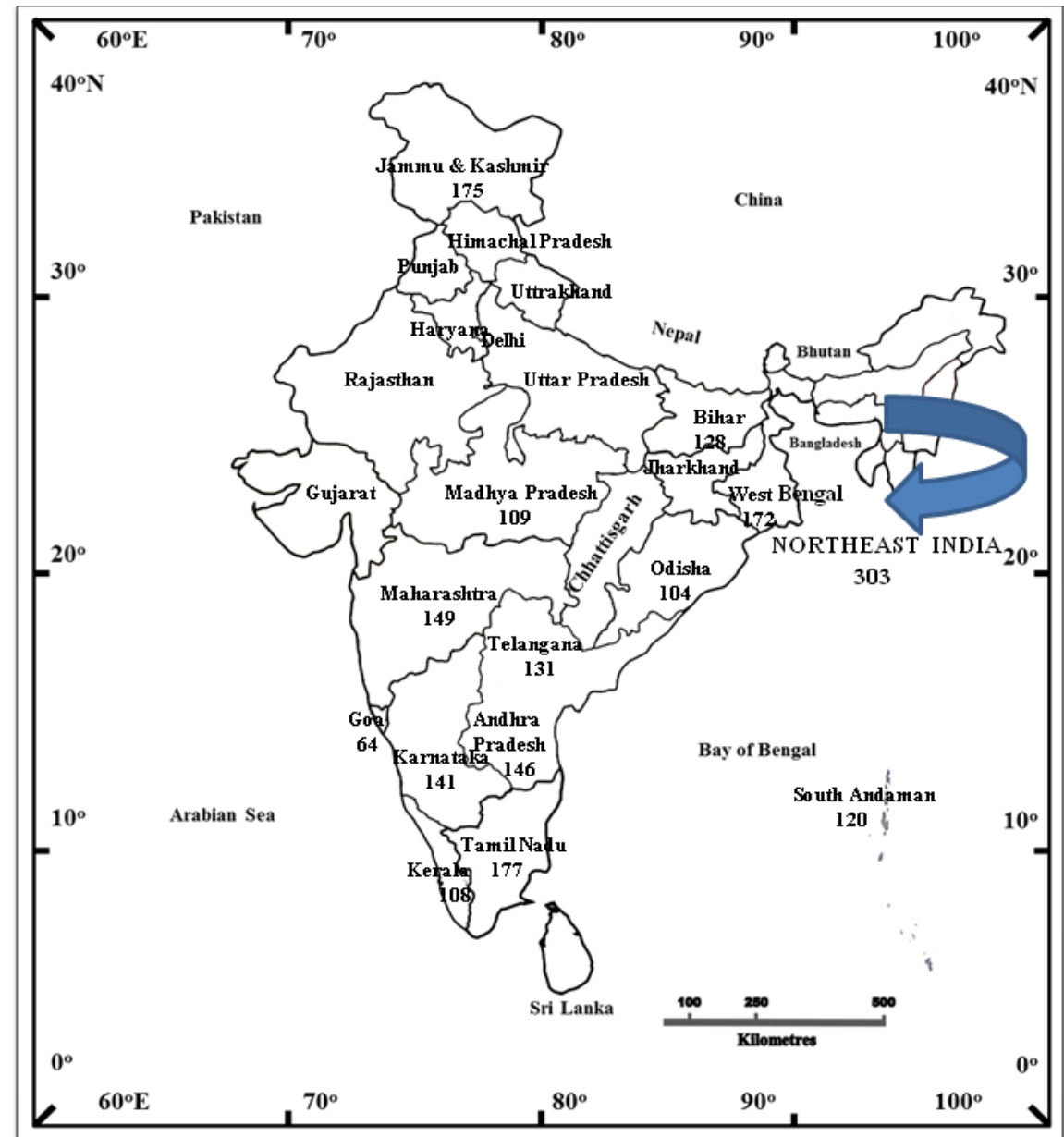

Figure 3. Species richness of Rotifera in various states of India

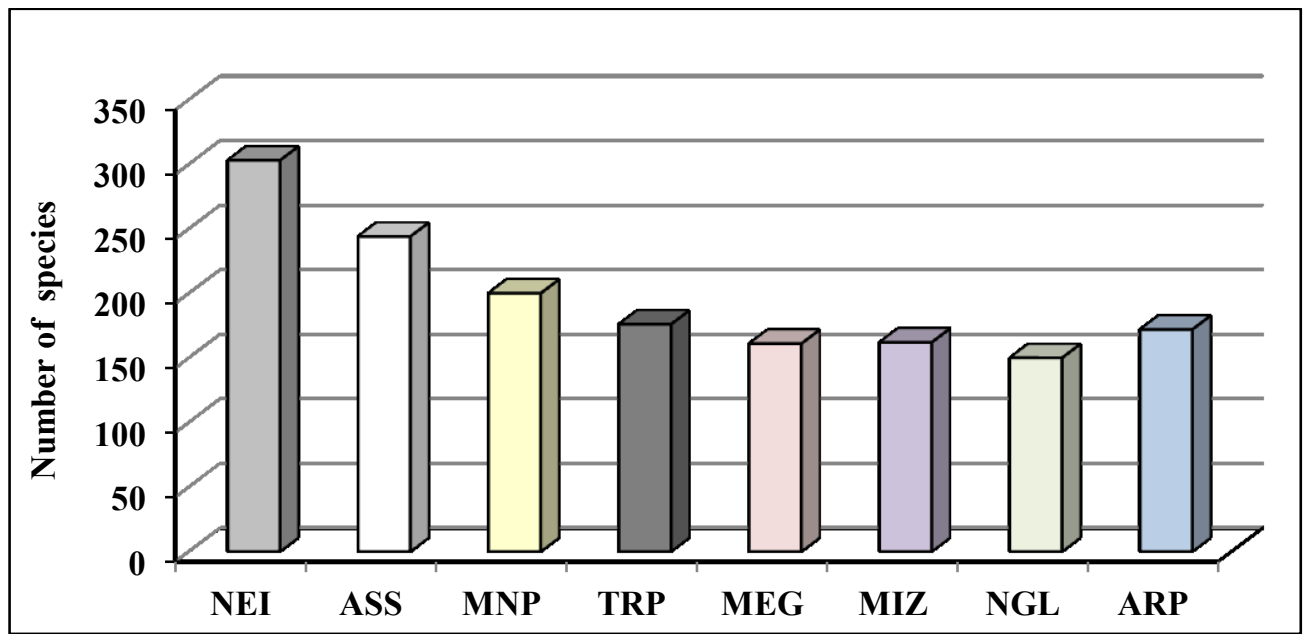

Figure 4. Rotifera species known from Northeast India

(NEI- northeast India; ASS-Assam; MNP-Manipur; TRP- Tripura; MEG-Meghalaya; MIZ-Mizoram, NGL-Nagaland; ARP-Arunachal Pradesh) 

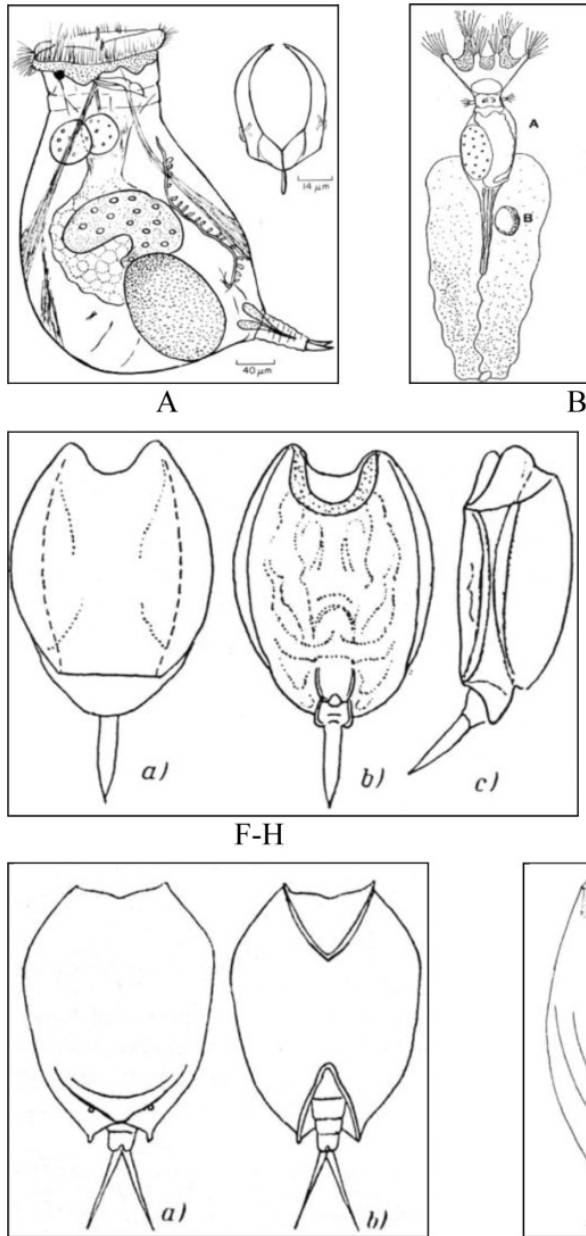

M-N

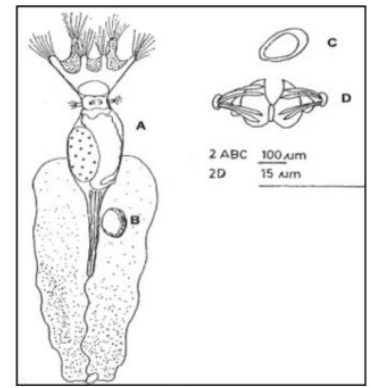

B

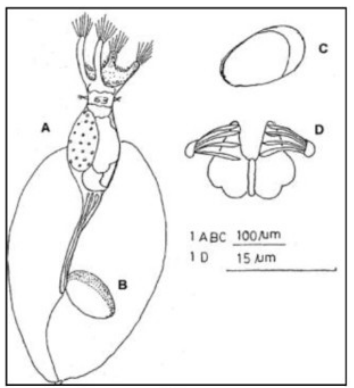

C

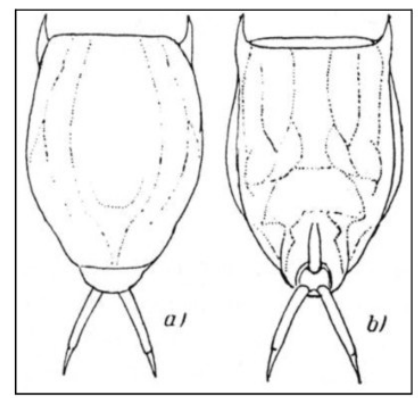

I-J

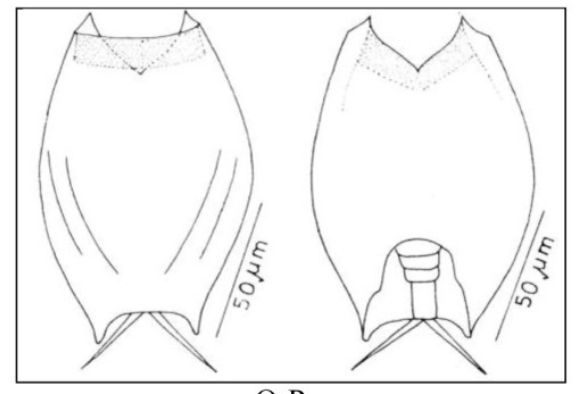

O-P

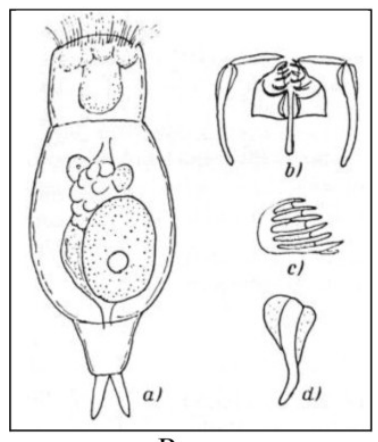

$\mathrm{R}$

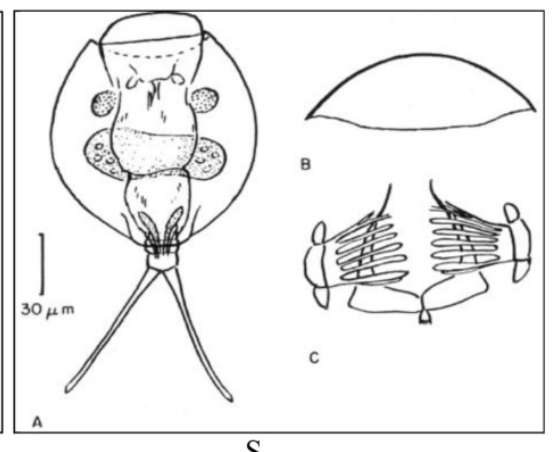

S

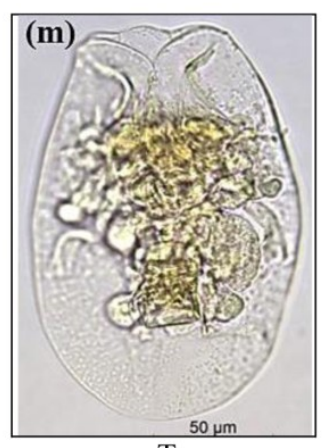

T

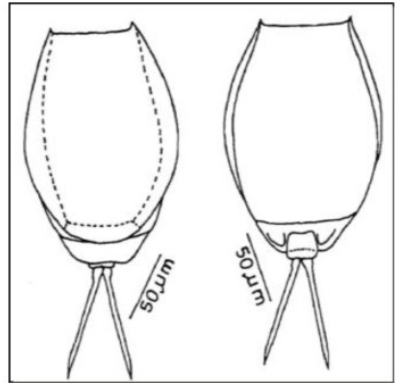

D-E

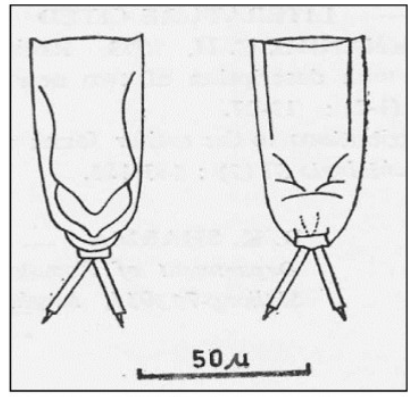

K-L

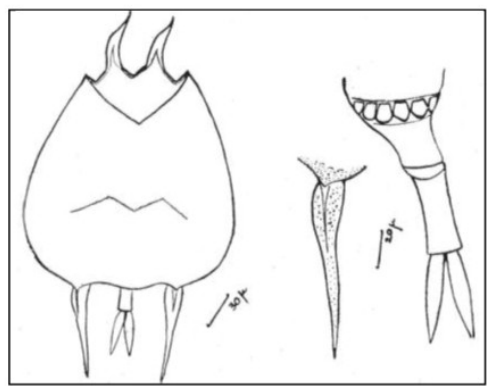

Q

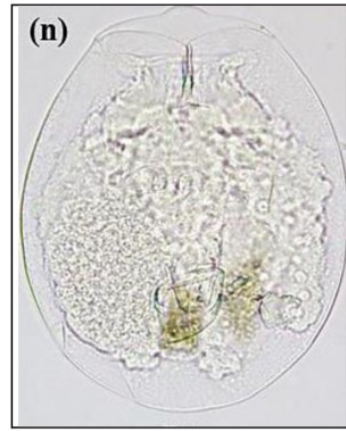

U

Figure 5. Indian endemics. $\mathbf{A}=$ Asplanchnopus bhimavaramensis Dhanapathi (lateral view and trophi, after Dhanapathi, 1975); $\mathbf{B}=$ Collotheca hexalobata Banik (lateral view, trophi and amictic egg, after Banik 2000); $\mathbf{C}=$ Collotheca tetralobata Banik (lateral view, trophi and amictic egg, after Banik 2000); D-E = Lecane jaintiaensis Sharma (dorsal and ventral view views, after Sharma 1987b); F-H = Lecane schraederi Wulfert (dorsal, ventral and lateral views); I-J = Lecane pawlowski Wulfert (dorsal and ventral views, after Wulfert, 1966); K-L = Lecane vasishti Sharma (dorsal and ventral view); M-N = Lepadella kostei Wulfert (dorsal and ventral views, after Wulfert 1966); O-P = Lecane nartiangensis Sharma \& Sharma (dorsal and ventral views, after Sharma \& Sharma 1987); Q = Platyias quadricornis andhraensis Dhanapathi (ventral view, after Dhanapathi, 1974); $\mathbf{R}=$ Proales indirae Wulfert (dorsal view, trophi, unci and ramu, after Wulfert 1966); $\mathbf{S}=$ Pseudoeuchlanis longipedes Dhanapathi (dorsal view, cross-section and trophi, after Dhanapathi 1978); $\mathbf{T}=$ Testudinella sp. (ventral view, after Sharma and Sharma 2018b); U = Testudinella sp.1 (ventral view, after Sharma \& Sharma 2018b). 


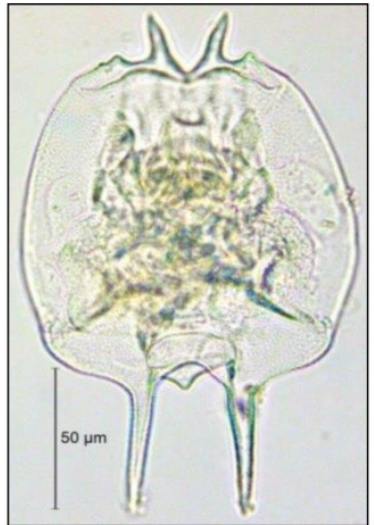

A

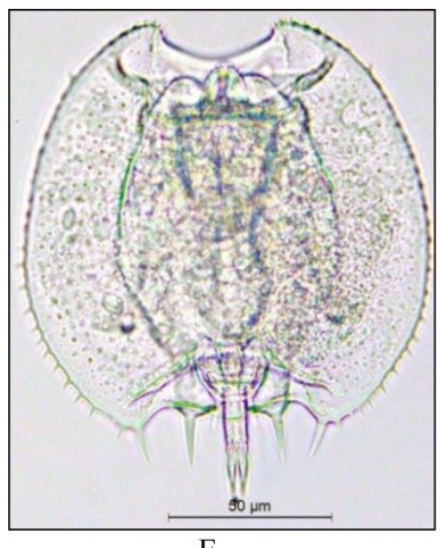

$\mathrm{E}$

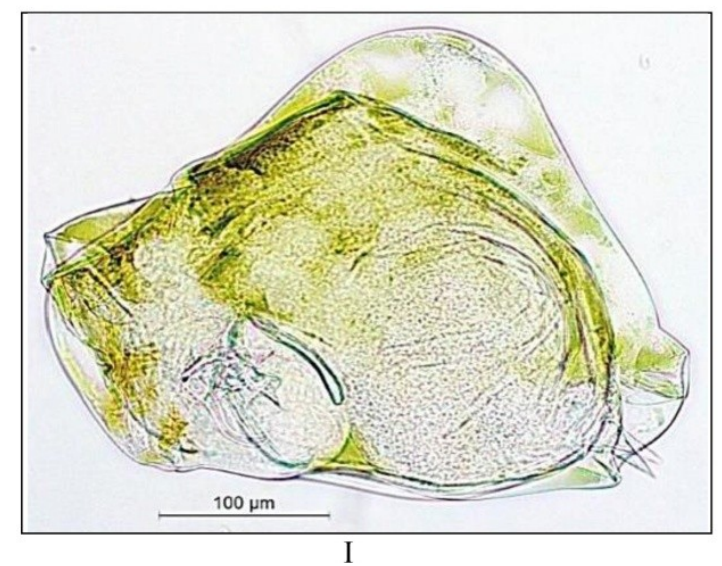

B

$\mathrm{F}$
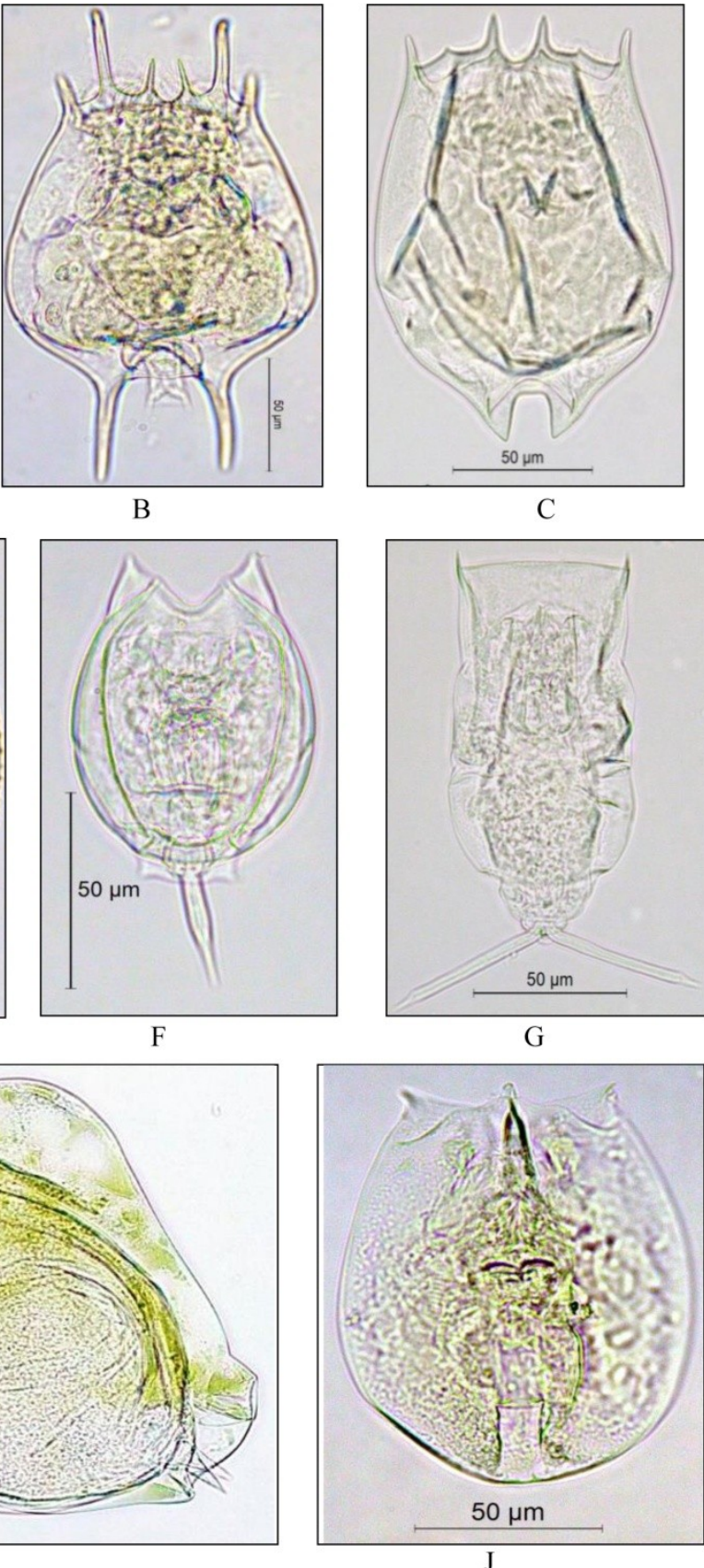

$\mathrm{J}$

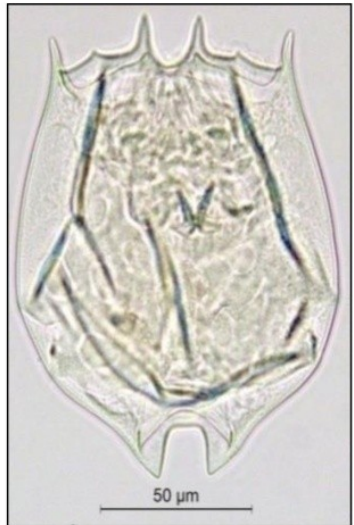

$\mathrm{C}$

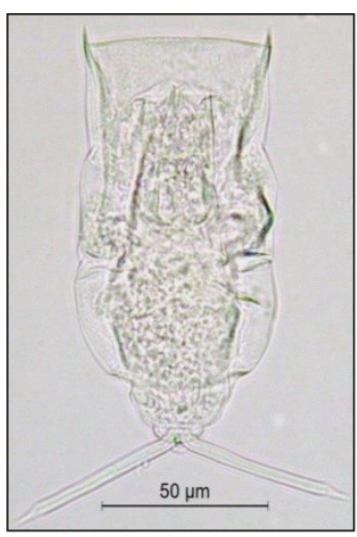

G

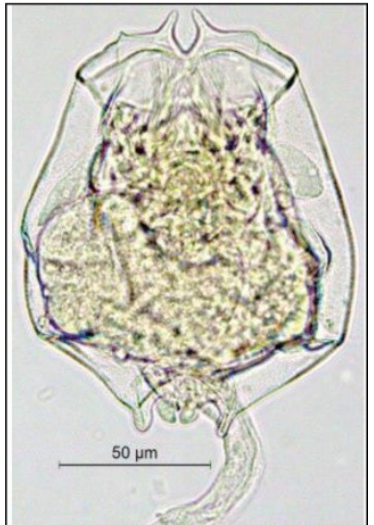

$\mathrm{D}$
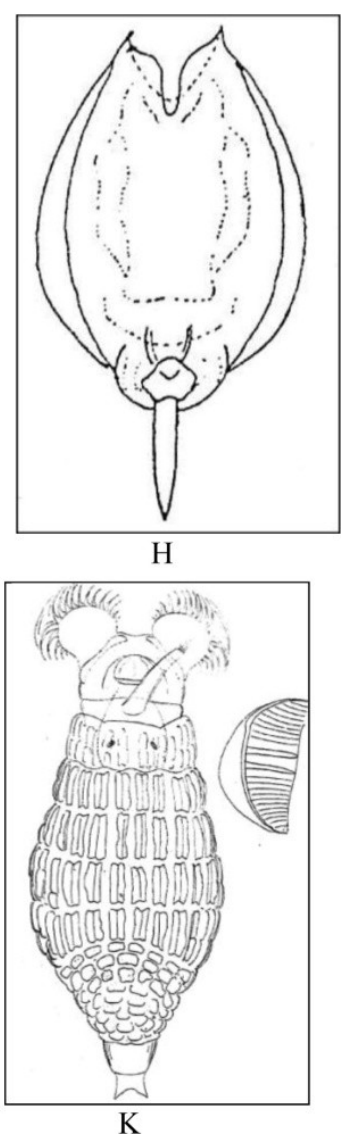

Figure 6. Australasian Rotifera. $\mathbf{A}=$ Brachionus dichotomus reductus Koste \& Shiel (ventral view); $\mathbf{B}=$ Brachionus falcatus reductus Koste \& Shiel (dorsal view, after Sharma \& Sharma 2019b); C = Brachionus kostei Shiel (dorsal view); D = Brachionus lyratus Shephard (ventral view, after Sharma \& Sharma 2019b); E = Macrochaetus danneelae Koste \& Shiel (dorsal view, after Sharma \& Sharma, 2019b); $\mathbf{F}=$ Lecane batillifer (Murray) (dorsal view); $\mathbf{G}=$ Lecane shieli Segers \& Sanoamuang (dorsal view, after Sharma \& Sharma 2019b); H = Lecane sinuata (Hauer) (ventral view, after Sharma \& Sharma 2014b); I = Notommata spinata Koste \& Shiel (parially compressed, lateral view); J = Testudinella walkeri Koste \& Shiel (ventral view, after Sharma \& Sharma 2015b); K = Philodina squamosa Murray (dorsal view and trophi, after Murray 1906). 

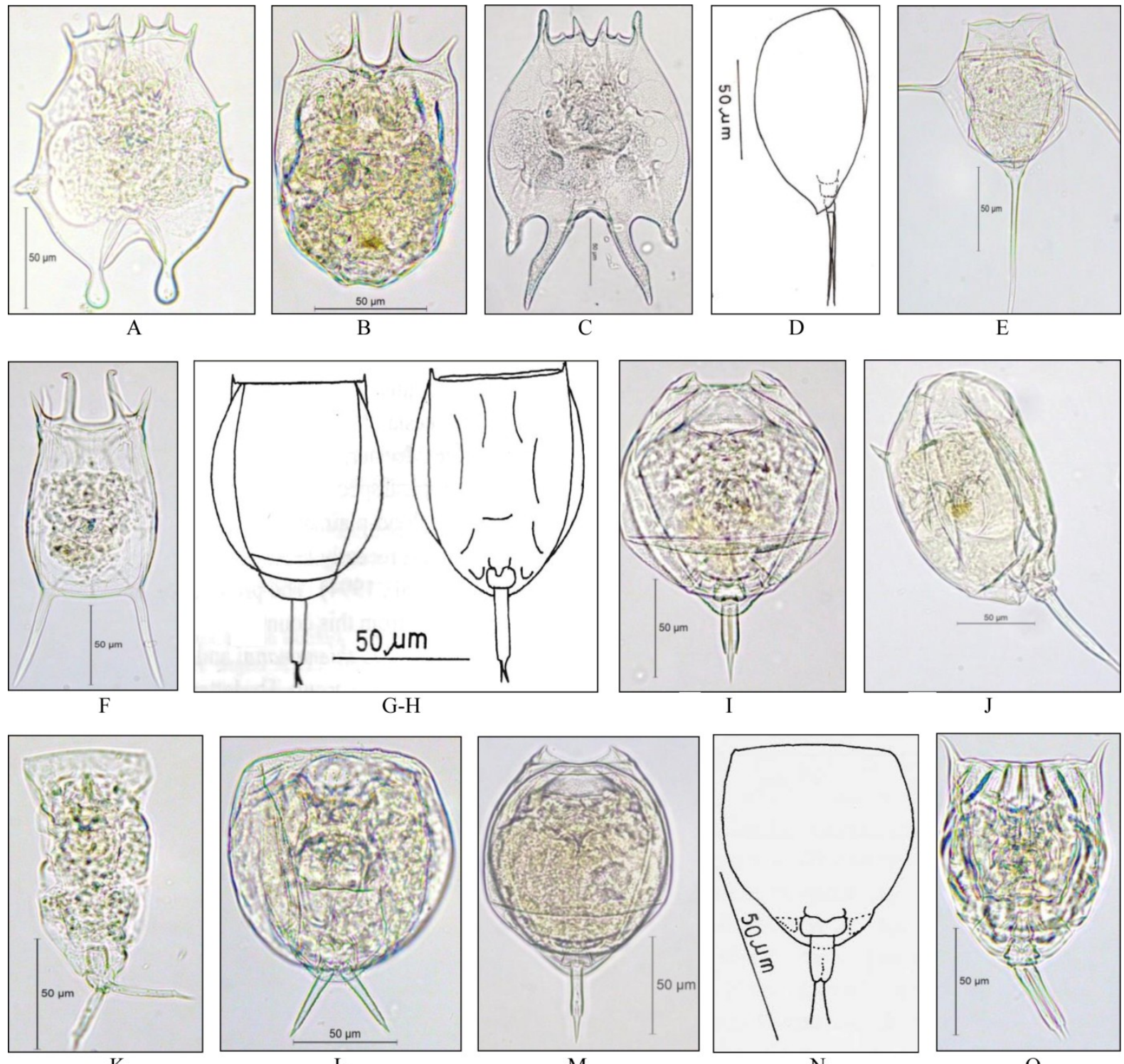

$\mathrm{L}$

M

$\mathrm{O}$

Figure 7. Oriental Rotifera. A = Brachionus donneri Brehm (ventral view); B = Brachionus murphyi Sudzuki (ventral view, after Sharma \& Sharma 2019b); C = Brachionus srisumonae Segers, Kothetip \& Sanoamuang (venral view, after Sharma \& Sharma 2019b); D = Colurella sanoamuangae Chittapun, Pholpunthin \& Segers (lateral view, after Sharma \& Sharma 2015a); E = Filinia camasecla Myers (ventral view); $\mathbf{E}=$ Keratella edmondsoni Ahlstrom (dorsal view); $\mathbf{G}-\mathbf{H}=$ Lecane acanthinula (Hauer) (dorsal and ventral views, after Sharma \& Sharma 2014b); I = Lecane blachei Berzins (ventral view); J = Lecane bulla diabolica (Hauer), lateral view; $\mathbf{K}=$ Lecane isanensis Sanoamuang \& Savatenalinton (ventral view, after Sharma \& Sharma 2019b); L = Lecane latissima Yamamoto (dorsal view); $\mathbf{M}=$ Lecane niwati Segers, Kothetip \& Sanoamuang (ventral view); $\mathbf{N}=$ Lecane solfatara (Hauer) (ventral view, after Sharma 2005); O = Lecane superaculeata Sanoamuang \& Segers (ventral view). 


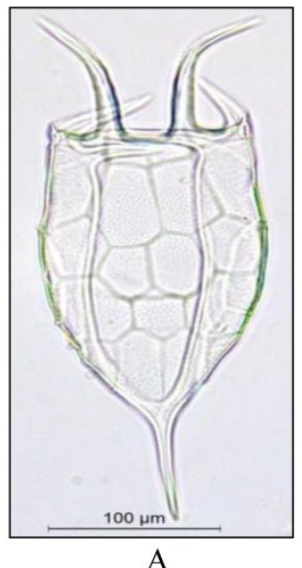

A

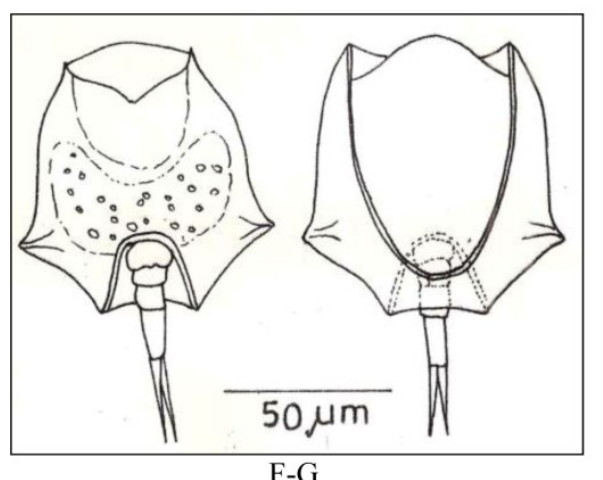

$\mathrm{F}-\mathrm{G}$

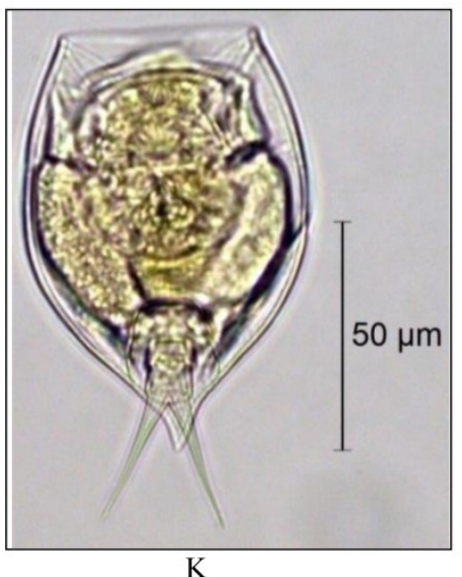

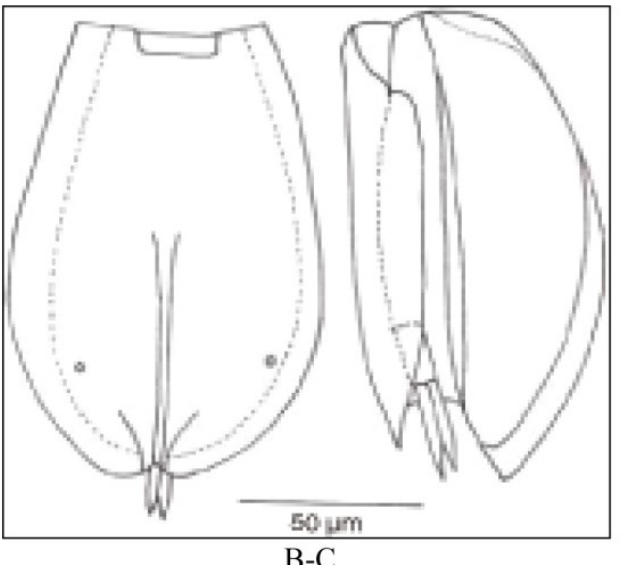

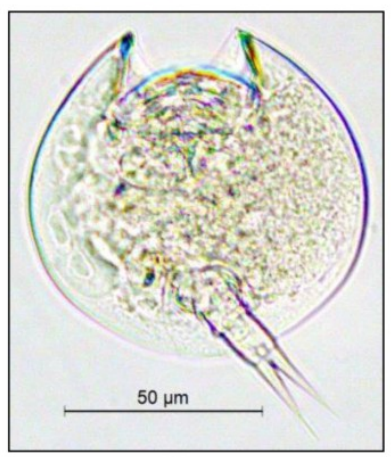

$\mathrm{H}$

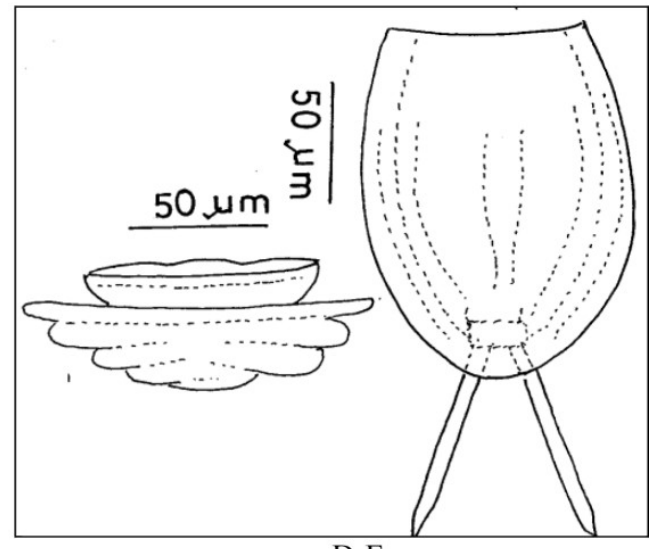

D-E

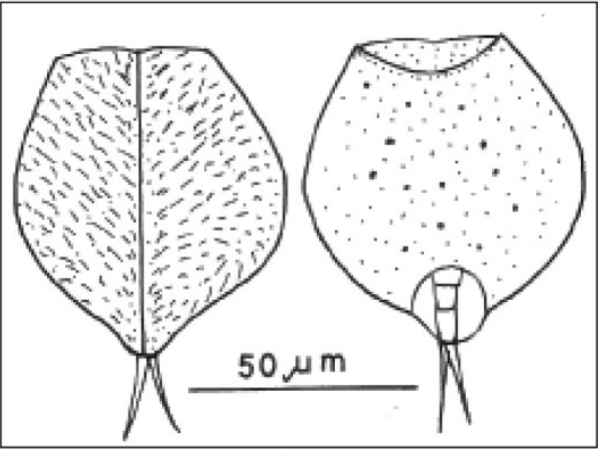

I-J

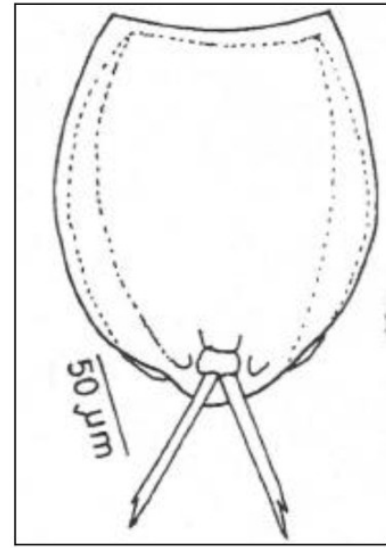

$\mathrm{L}$

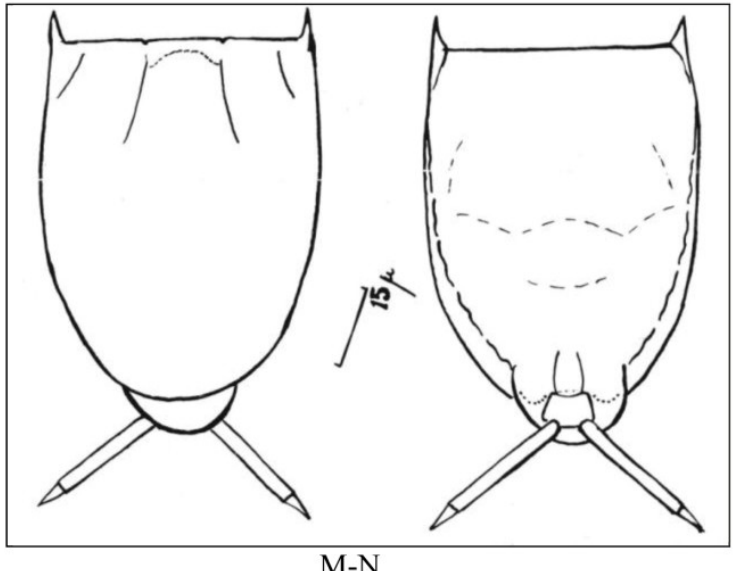

$\mathrm{M}-\mathrm{N}$

Figure 8. Paleotropical Rotifera. A = Keratella javana Hauer (ventral view); B-C = Euchlanis semicarinata Segers(dorsal and lateral views, after Sharma 2005); D-E = Dipleuchlanis ornata Segers (ventral view and cross-section, after Sharma 2005); F-G = Lecane bicornis Vasisht \& Battish (ventral and dorsal views); H = Lepadella discoidea Segers (ventral view); I-J = Lepadella minoruoides Koste \& Robertson (dorsal and ventral view, after Sharma 2004); K=Lepadella vandenbrandei Gillard (ventral view); $\mathbf{L}=$ Lecane braumi, Koste (ventral view, after Sharma \& Sharma 1987); M-N = Lecane eswari Dhanapathi (dorsal and ventral views, after Dhanapathi 1976 ) 


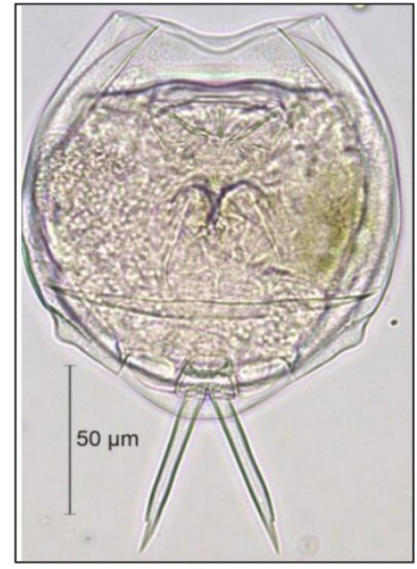

A

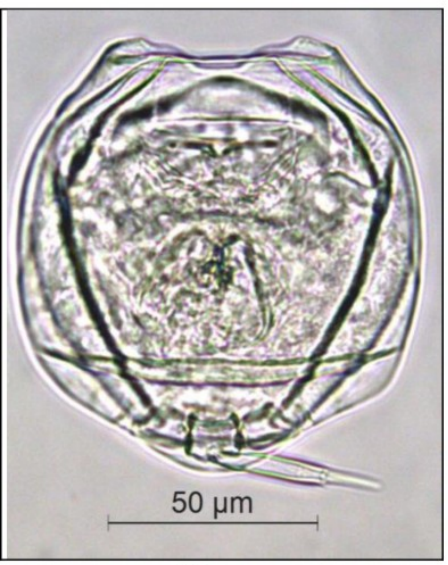

$\mathrm{D}$

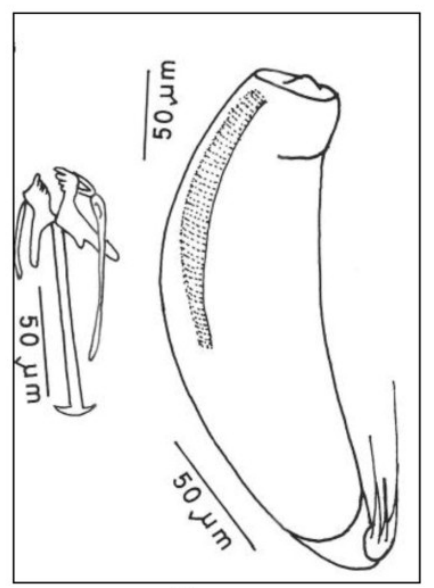

G

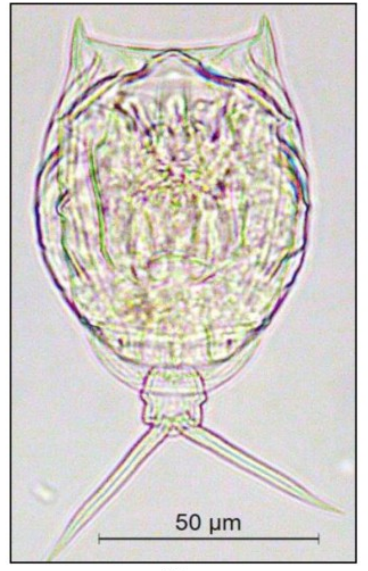

B

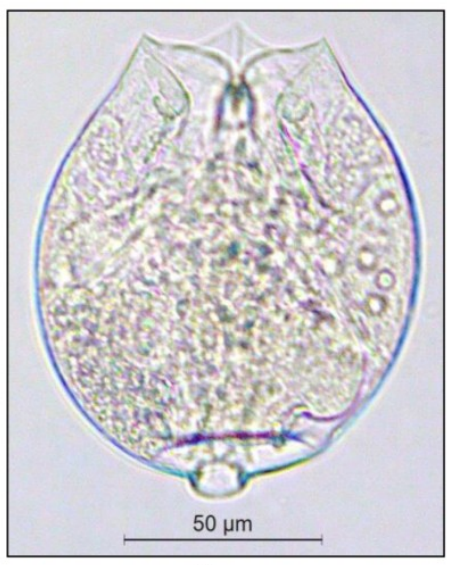

E

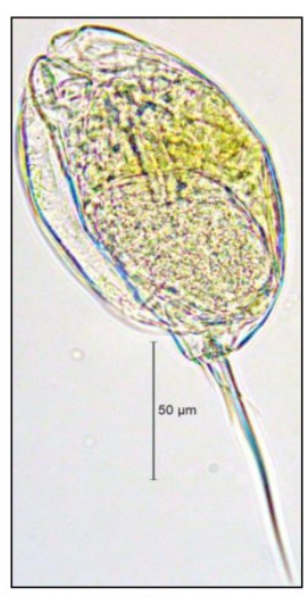

$\mathrm{H}$

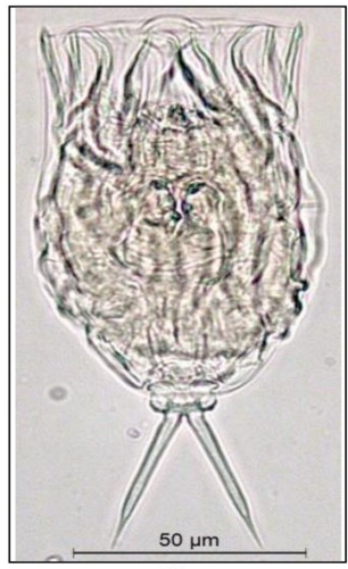

C

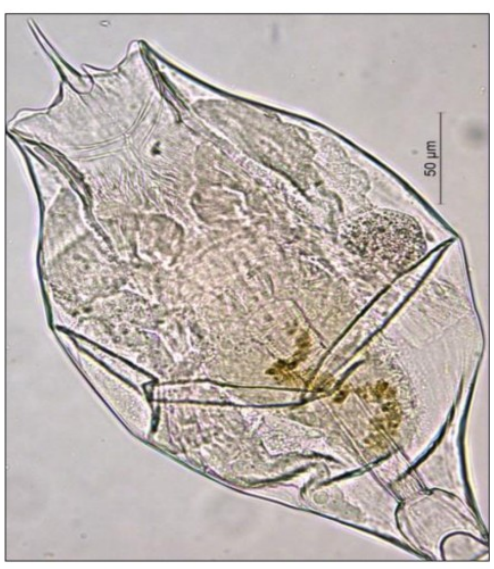

$\mathrm{F}$

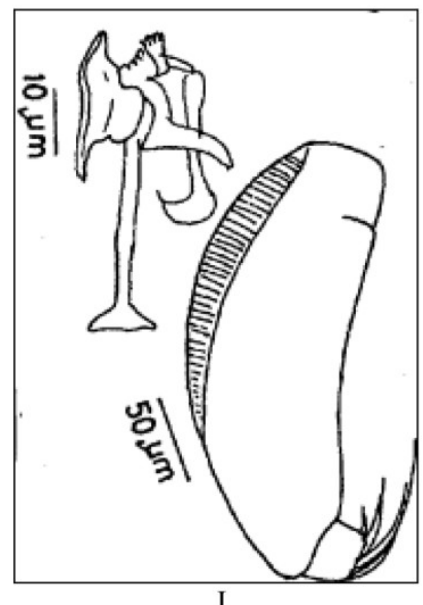

Figure 9. Paleotropical Rotifera. $\mathbf{A}=$ Lecane lateralis Sharma, (ventral view); $\mathbf{B}=$ Lecane simonneae Segers (dorsal view); $\mathbf{C}=$ Lecane stichoclysta Segers (dorsal view, Sharma \& Sharma 2019c); D = Lecane unguitata (Fadeev) (ventral view);

$\mathbf{E}=$ Testudinella brevicaudata Yamamoto, ventral view; $\mathbf{F}=$ Testudinella greeni Koste (dorsal view);

$\mathbf{G}=$ Trichocerca abilioi Segers \& Sarma (lateral view and trophi, Sharma \& Sharma 2008);

$\mathbf{H}=$ Trichocerca hollaerti De Smet (lateral view); $\mathbf{I}=$ Trichocerca kostei Segers (lateral view and trophi, Sharma \& Sharma, 2008). 

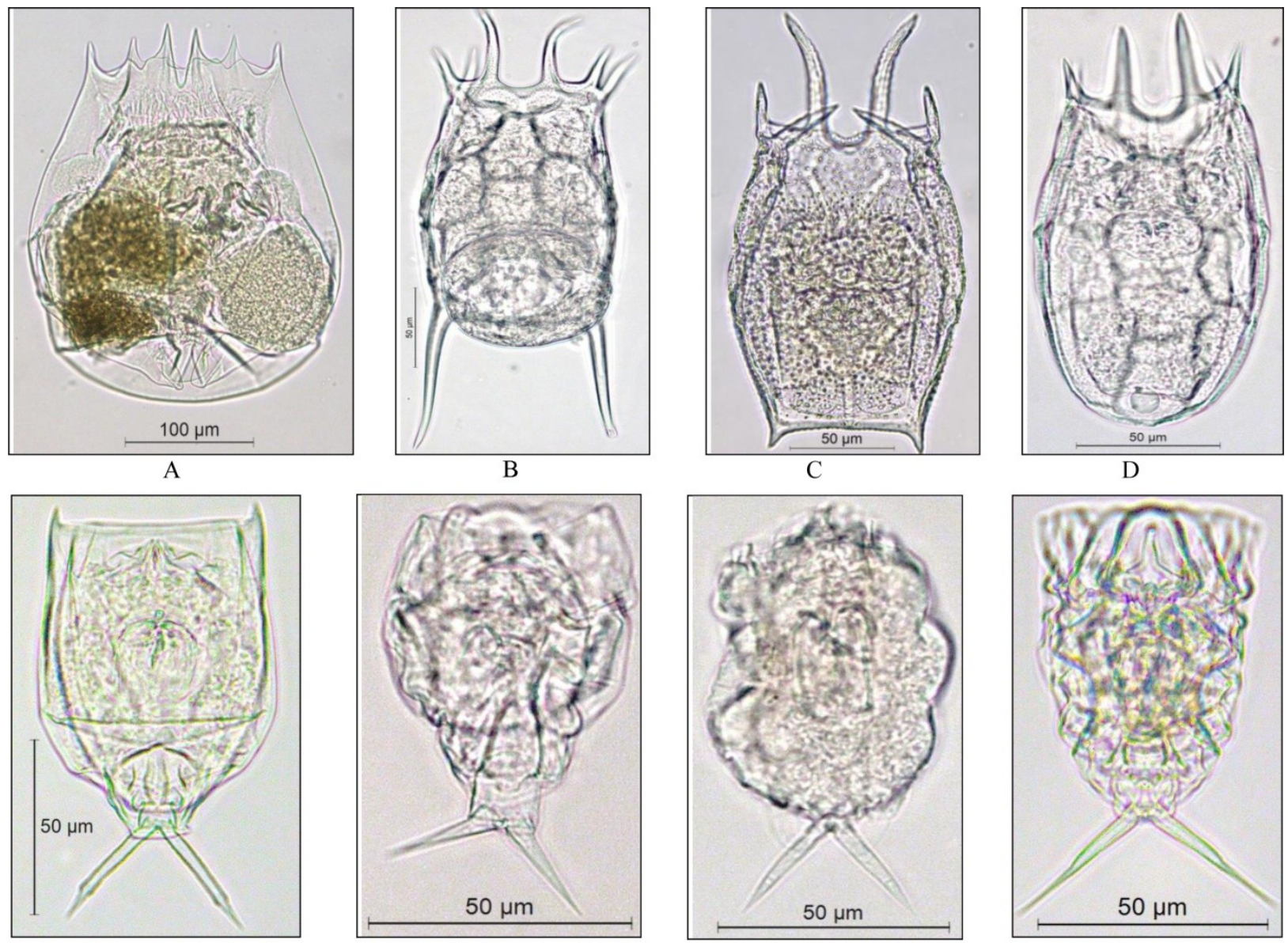

$\mathrm{E}$
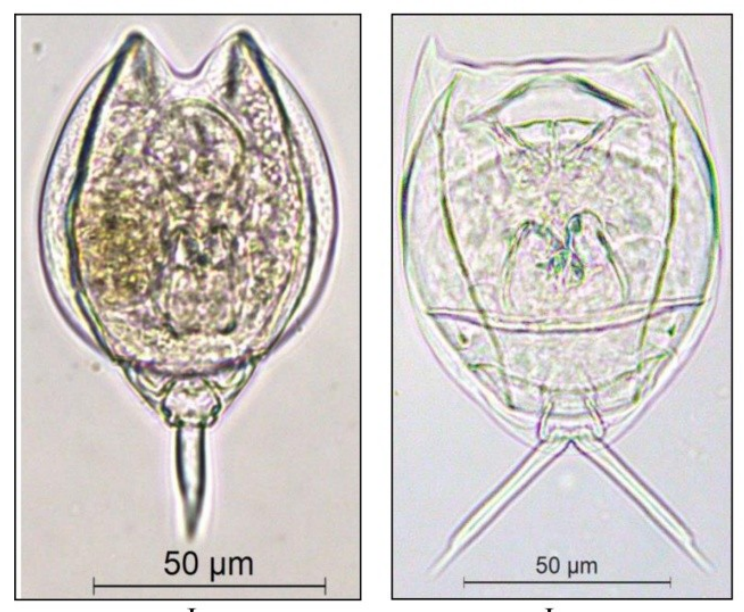

G

$\mathrm{H}$

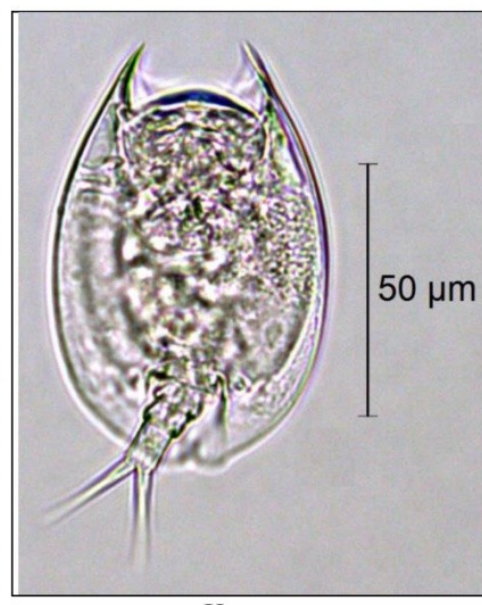

K

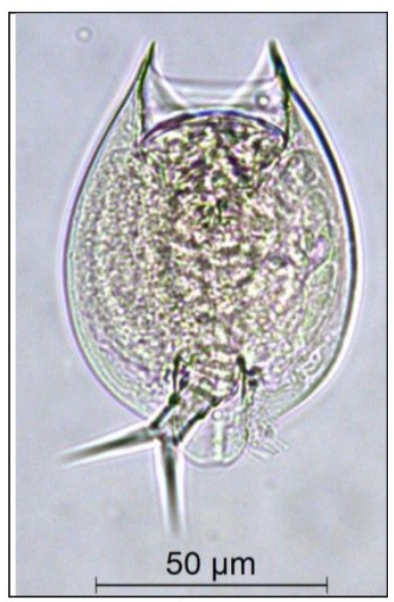

$\mathrm{L}$

Figure 10. Interesting species. $\mathbf{A}=$ Brachionus durgae Dhanapathi (dorsal view); $\mathbf{B}=$ Keratella hiemalis Carlin (ventral view, Sharma \& Sharma 2018a); C = Keratella serrulata (Ehrenberg) (dorsal view, Sharma \& Sharma 2018a); D = Keratella ticinensis (Callerio) (ventral view, Sharma and Sharma 2018a); $\mathbf{E}=$ Lecane bifastigata Hauer, (ventral view); $\mathbf{F}=$ Lecane calcaria Harring \& Myers (ventral view); $\mathbf{G}=$ Lecane clara (Bryce) (dorsal view); $\mathbf{H}=$ Lecane dorysimilis Trinh Dang, Segers \& Sanoamuang (ventral view); I = Lecane marchantaria Koste \& Robertson (dorsal view, Sharma \& Sharma 2019a); J = Lecane rhenana Hauer (ventral view); $\mathbf{K}=$ Lepadella desmeti Segers \& Chittapun (ventral view, Sharma \& Sharma 2015a); $\mathbf{L}=$ Lepadella neglecta Segers \& Dumont (ventral view, Sharma \& Sharma 2018c). 


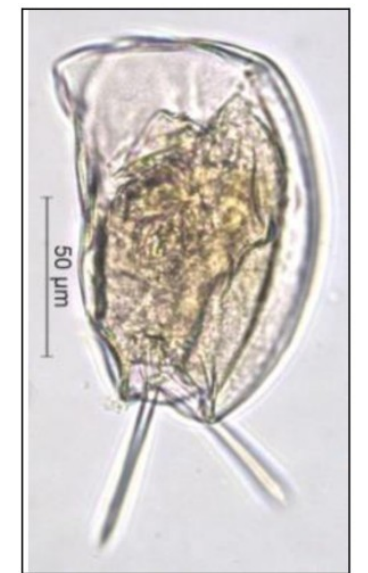

A

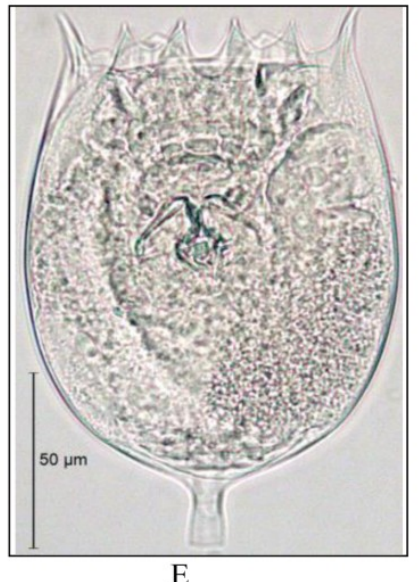

E

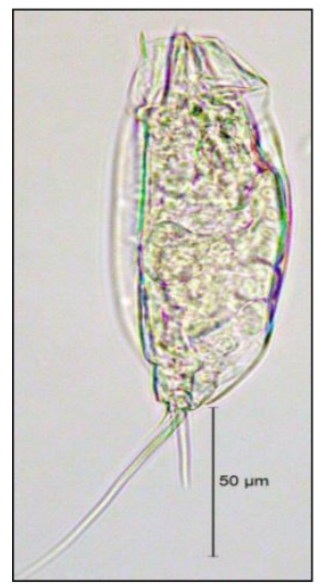

$\mathrm{H}$

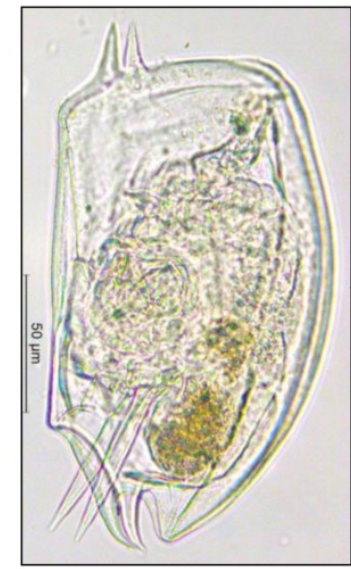

B

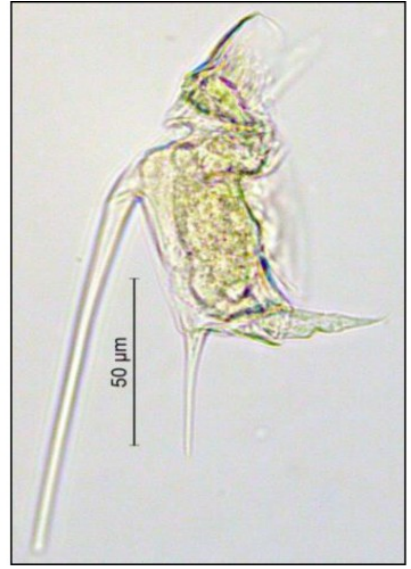

C

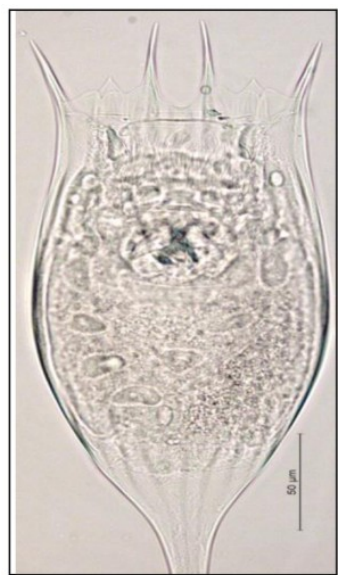

D

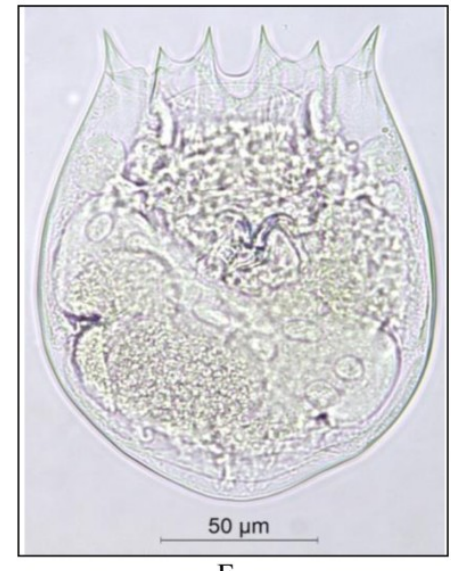

F

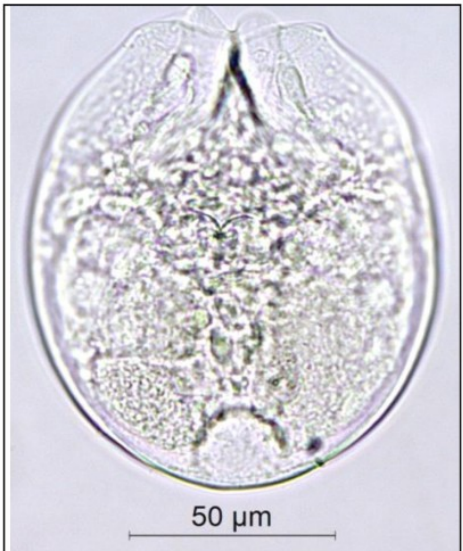

G

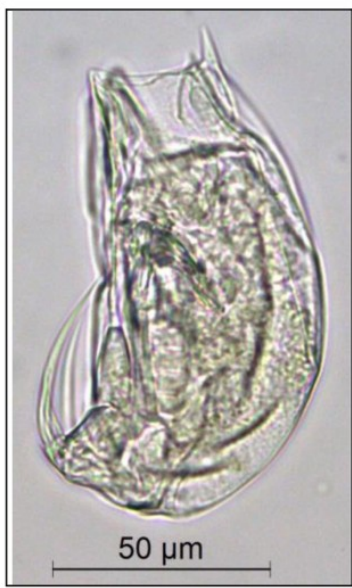

I

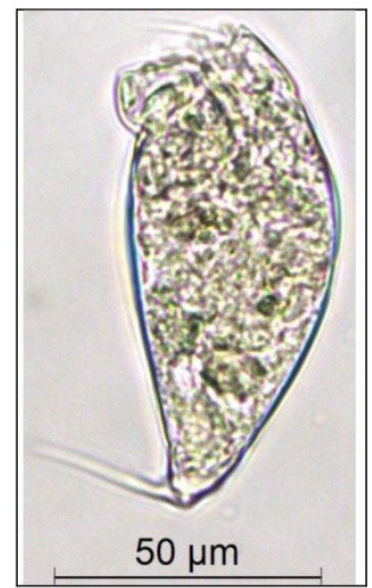

$\mathrm{J}$

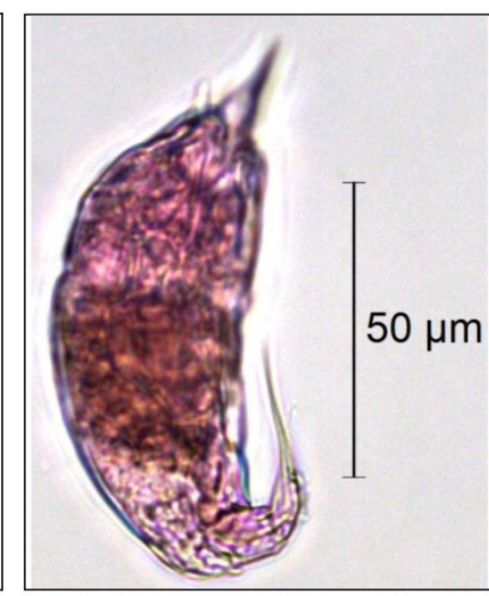

$\mathrm{K}$

Figure 11. Interesting Rotifera. A = Mytilina lobata Pourriot (lateral view, Sharma \& Sharma 2019b); B = Mytilina michelangellii Reid \& Turner (lateral view); C = Squatinella bifurca (Bolton) (lateral view, Sharma et al. 2017); D = Notholca acuminata (Ehrenberg) (ventral view, Sharma \& Sharma 2018c); $\mathbf{E}=$ Notholca labis Gosse (ventral view, Sharma \& Sharma 2018c); $\mathbf{F}=$ Notholca squamula (Muller) (ventral view); G = Testudinella insinuata Hauer (ventral view, Sharma and Sharma, 2018b); H = Trichocerca edmondsoni (Myers) (lateral view); $\mathbf{I}=$ Trichocerca maior Hauer (lateral view); $\mathbf{J}=$ Trichocerca siamensis Segers \& Pholpunthin (lateral view, Sharma \& Sharma, 2015a); K = Trichocerca taurocephala (Hauer) (lateral view, after Sharma \& Sharma 2015a). 
Indian Rotifera includes 11 Australasian, 15 Oriental, 20 Paleotropical, 15 Indian endemics, 10 Holarctic and four Palearctic species, one species each of the Indo-Chinese and Cosmo (sub) tropical categories, eight cold-water species and 16 other interesting species (Figs. 5-11). A total of 176 species depict regional distribution interest in India; of these, 70 species are known for their distribution restricted to NEI India. The rotifer diversity in Ramsar sites, floodplain lakes of Assam (beels) and Manipur (pats) and small floodplain wetlands (dobas or dubies) and small lentic biotopes of NEI; the floodplain wetlands of Kashmir and West Bengal; and small lentil environs of the Gangetic and north Bengal regions of West Bengal are included in Table 2.

Table 2: Rotifera richness in various aquatic ecosystems (Modified after Sharma \& Sharma 2019b)

\begin{tabular}{|c|c|c|c|}
\hline Study sites $\downarrow$ & Species & Genera & Families \\
\hline \multicolumn{4}{|l|}{ NORTHEAST INDIA } \\
\hline \multicolumn{4}{|l|}{ Ramsar sites } \\
\hline Loktak Lake, Manipur $\left(93^{\circ} 46^{\prime}-93^{\circ} 55^{\prime} \mathrm{E}, 24^{\circ} 25^{\prime}-24^{\circ} 42^{\prime} \mathrm{N}\right)$ & 203 & 48 & 23 \\
\hline Deepor Beel, Assam $\quad\left(91^{\circ} 35^{\prime}-91^{\circ} 43^{\prime} \mathrm{E}, 26^{\circ} 05^{\prime}-26^{\circ} 11^{\prime} \mathrm{N}\right)$ & 183 & 36 & 20 \\
\hline Floodplain lakes (beels) of Brahmaputra Basin, Assam & 244 & 46 & 21 \\
\hline Barpeta (6 beels $) \quad\left(90^{\circ} 52^{\prime}-91^{\circ} 42^{\prime} \mathrm{E}, 26^{\circ} 17^{\prime}-26^{\circ} 40^{\prime} \mathrm{N}\right)$ & 176 & 35 & 19 \\
\hline Majuli River Island $(10$ beels $)\left(93^{\circ}-95^{\circ} \mathrm{E}, 25^{\circ}-27^{\circ} \mathrm{N}\right)$ & 174 & 34 & 18 \\
\hline $\begin{array}{l}\text { Dibru-Saikhowa Biosphere Reserve (5 beels) } \\
\qquad\left(95^{\circ} 22^{\prime}-95^{\circ} 24^{\prime} \mathrm{E}, 27^{\circ} 34^{\prime}-27^{\circ} 55^{\prime} \mathrm{N}\right)\end{array}$ & 162 & 32 & 18 \\
\hline Tinsukia (5 beels $) \quad\left(95^{\circ} 22^{\prime}-96^{\circ} 35^{\prime} \mathrm{E}, 27^{\circ} 14^{\prime}-28^{\circ} 40^{\prime} \mathrm{N}\right)$ & 169 & 33 & 19 \\
\hline$\left(93^{\circ} 22^{\prime}-95^{\circ} 35^{\prime} \mathrm{E}, 26^{\circ} 19^{\prime}-27^{\circ} 30^{\prime} \mathrm{N}\right)$ & 179 & 35 & 19 \\
\hline \multicolumn{4}{|l|}{ Floodplain lakes (pats) of Manipur } \\
\hline Manipur valley $(15$ pats $)\left(93^{\circ} 45^{\prime}-94^{\circ} 00^{\prime} \mathrm{E}, 24^{\circ} 25^{\prime}-24^{\circ} 45^{\prime} \mathrm{N}\right)$ & 218 & 48 & 23 \\
\hline \multicolumn{4}{|l|}{ Small floodplain wetlands (dobas or dubies) of Assam } \\
\hline Brahmaputra valley $\quad\left(90^{\circ}-93^{\circ} \mathrm{E} ; 26^{\circ}-27^{0} \mathrm{~N}\right)$ & 167 & 34 & 18 \\
\hline Lower Assam & 154 & 34 & 18 \\
\hline Central Assam & 150 & 31 & 19 \\
\hline Upper Assam & 135 & 30 & 17 \\
\hline$\left(92^{\circ} 45^{\prime}-92.75^{\circ} \mathrm{E}, 24^{\circ} 48^{\prime}-24.80^{\circ} \mathrm{N}\right)$ & 159 & 35 & 19 \\
\hline \multicolumn{4}{|l|}{ NEI: small lentic ecosystems } \\
\hline Arunachal Pradesh $\quad\left(91^{\circ} 20^{\prime}-97^{\circ} 30^{\prime} \mathrm{E}, 26^{\circ} 28 \mathrm{v}-29^{\circ} 30^{\prime} \mathrm{N}\right)$ & 165 & 37 & 19 \\
\hline$\left(93^{\circ} 3^{\prime}-93^{\circ} 5^{\prime} \mathrm{E}, 25^{\circ} 4^{\prime}-27^{\circ} 0^{\prime} \mathrm{N}\right)$ & 150 & 37 & 19 \\
\hline$\left(92^{\circ} 15^{\prime}-93^{\circ} 29^{\prime} \mathrm{E}, 21^{\circ} 58^{\prime}-24^{\circ} 35^{\prime} \mathrm{N}\right)$ & 162 & 35 & 19 \\
\hline$\left(90^{\circ} 05^{\prime}-92^{\circ} 40^{\prime} \mathrm{E}, 25^{\circ} 10^{\prime}-26^{\circ} 15^{\prime} \mathrm{N}\right)$ & 161 & 40 & 20 \\
\hline$\left(92^{\circ} 10^{\prime}-92^{\circ} 20^{\prime} \mathrm{E}, 22^{\circ} 56^{\prime}-24^{\circ} 32^{\prime} \mathrm{N}\right)$ & 163 & 35 & 19 \\
\hline Manipur $\quad\left(93^{\circ} 50^{\prime}-94^{\circ} 00^{\prime} \mathrm{E}, 2^{\circ} 10^{\prime}-24^{\circ} 55^{\prime} \mathrm{N}\right)$ & 169 & 44 & 22 \\
\hline Meghalaya: small urban wetland & 90 & 29 & 15 \\
\hline \multicolumn{4}{|l|}{ OTHERS PARTS OF INDIA } \\
\hline Floodplain wetlands of Kashmir valley & 140 & 43 & 22 \\
\hline Floodplain wetlands of West Bengal & 152 & 40 & 19 \\
\hline Small lentic ecosystems of Gangetic West Bengal & 142 & 38 & 18 \\
\hline Small lentic ecosystems of north Bengal & 130 & 40 & 18 \\
\hline
\end{tabular}




\section{DISCUSSION}

We record a total of 434 valid species belonging to 68 genera and 25 families; these comprise $\sim 82 \%$ and $\sim 24 \%$ of the species of the phylum known from the Oriental region and globally (vide Segers 2008), respectively, and thus reveal the rich and diverse Rotifera assemblage of India. The Indian fauna is more speciose than the faunas of Thailand (Sa-Ardrit et al. 2013), Cambodia (Sor et al. 2015), Vietnam (Trinh et al. 2019), and Malaysia (Segers 2004, Fontaneto \& Ricci 2004) from SE Asia, and is distinctly diverse than the fauna of Sri Lanka (Fernando 1980) - the sole reasonably studied country of south Asia. The comparisons characterize the Indian Rotifera to be most biodiverse vis-a-vis south and SE Asia faunas. Besides, a total of 359 species belonging to 25 families and 67 genera observed in our plankton and semi-planktonic collections from various regions comprise $\sim 81 \%, \sim 88 \%$ and $96 \%$ of species, genera and families, respectively known from this country and thus reiterate important contributions of our studies to the rotifer fauna of India. In general, the Indian faunal surveys lacked focus on biodiversity till the end of the $20^{\text {th }}$ century, while it received attention in limited subsequent works to-date. Nevertheless, the significant increase in the tally of rotifers species known from India, than the earlier reports (Sharma \& Michael 1980, Sharma 1991, 1996, 1998a, Sharma \& Sharma 2005), is attributed notably to our studies from NEI (Sharma 2004, 2005, 2014, Sharma \& Sharma 2012, 2014a, 2014b, 2014c, 2015a, 2015b, 2015c, 2018a, 2018b, 2019a, 2019b, 2019c, 2019d, Sharma et al. 2016, 2017) and Jammu \& Kashmir (Sharma \& Sharma 2018a), and elsewhere from Madhya Pradesh (Sharma \& Naik 1996), Tamil Nadu (Sharma \& Sharma 2009) and West Bengal (Sharma 1998b).

Lecanidae $(21.5,27.9 \%)>$ Brachionidae $(11.8$, $15.4 \%)>$ Lepadellidae $(10.4,13.5 \%)$ contribute notably to the rotifer and monogonont species known to-date from India, respectively; the three families collectively comprise an important fraction $(\sim 44 \%)$ of the Indian Rotifera. In addition,
Trichocercidae $>$ Notommatidae $>$ Philodinidae include $\sim 22 \%$ species, while Floscularidae $>$ Testudinellidae $>$ Euchlanidae $>$ Synchaetidae $=$ Trochosphaeridae indicate limited importance $(\sim 14 \%)$. The significance of the stated families imparts the 'littoral-periphytic' character to the Indian Rotifera broadly concurrent with the reports from Thailand (Sa-Ardrit et al. 2013), Cambodia (Sor et al. 2015) and Vietnam (Trinh et al. 2019). This generalization is further supported by inadequate documentation of the bdelloid and sessile rotifers from India till-date. The latitudinal variations of Rotifera vs. biogeographic role of the 'tropic' or 'temperate' centered taxa had been discussed by Green (1972), Pejler (1977), De Ridder (1981), Dumont (1983) and Segers (1996, 2001). We extend these remarks to the Indian rotifers vides the diverse nature of Lecanidae, Brachionidae and Lepadellidae, the speciose nature of the 'tropic-centered' Lecane, Lepadella and Brachionus (Sharma \& Sharma 2017, 2019b) and also to certain extent that of 'Laurasian' centered Trichocerca. These features along with the predominance of cosmopolitan species and the reports of several pantropical and cosmotropical species impart a broadly 'tropical character' to the Indian Rotifera in agreement with the reports of Fernando (1980), Dussart et al. (1984), Segers (1996, 2001, 2008) and Green (2003). On the contrary, the localized valid reports of cold-water species of 'temperate' centered' Kellicottia, Keratella, Synchaeta and Notholca from the subHimalayan and Himalayan latitudes of India are diagnostic of specific ecological regimes. We caution on emphasis of 'cosmopolitan species' importance as 'cosmopolitanism' concept is debated in certain freshwater zooplankton groups vides the integrative taxonomical approaches.

Rotifera of India is characterized by a notable fraction $(\sim 25 \%)$ of species of the global biogeography interest; these are assigned to the following categories:

Australasian: Brachionus dichotomus reductus, $B$. falcatus reductus, B. kostei, B. lyratus, Macrochaetus danneelae, Lecane batillifer, L. shieli, L. sinuata, Notommata spinata, Testudinella walkeri, and Philodina squamosa; 
Oriental: Brachionus donneri, B. murphyi, B. srisumonae, Colurella sanoamuangae, Filinia camasecla, Keratella edmondsoni, Lecane acanthinula, L. blachei, L. bulla diabolica, L. isanensis, L. latissima, L. niwati, L. solfatara, L. superaculeata, and Ptygura stephanion;

Paleotropical: Keratella javana, Dipleuchlanis ornata, Euchlanis semicarinata, Lepadella bicornis, L. discoidea, L. minoruoides, L. vandenbrandei, Lecane braumi, L. eswari, L. lateralis, L. simonneae, L. stichoclysta, L. unguitata, Polyarthra indica, Testudinella brevicaudata, T. greeni, Trichocerca abilioi, T. brazieliensis, T. hollaerti, and T. kostei;

Indian endemics: Asplanchnopus bhimavaramensis, Collotheca hexalobata, C. tetralobata, Lecane jaintiaensis, L. schraederi, L. pawlowski, L. vasishti, Lepadella kostei L. nartiangensis, Platyias quadricornis andhraensis, Proales indirae, Pseudoeuchlanis longipedes, Rotaria ovata, Testudinella sp., and T. sp. 1 (undetermined vide Sharma \& Sharma 2018b);

Holarctic: Lecane depressa, L. elasma, L. elongata, L. galeata, L. levistyla, L. stokesii), L. styrax, L. tryphema, Trichocerca taurcephala, and T. uncinata;

Palaearctic: Encentrum longipes, Cephalodella trigona, Lecane bifastigata, and Squatinella bifurca;

Indo-Chinese: Lecane dorysimilis;

Cosmo (sub) tropical: Brachionus durgae;

Cold-water: Hexarthra bulgarica, Keratella hiemalis, K. serrulata, Kellicottia longispina, Notholca acuminata, N. labis, N. squamula, and N. striata,

Others: Lecane calcaria, L. ligona, L. marchantaria, L. rhenana, L. ruttneri, L rugosa, L. sola, Lepadella desmeti, L. patella oblonga, L. neglecta, Mytilina lobata, M. michelangellii, Ptygura tacita, Testudinella amphora, Trichocerca edmondsoni, and T. siamensis.

The reports of the Australasian rotifers, known exclusively from south and SE Asia and Australia, highlight affinity of the Indian Rotifera with Southeast Asian and Australian faunas. The Oriental rotifers reiterate affinity with south and Southeast Asia faunas, and the richness of Pale- otropical species is noteworthy. Interestingly, the reports of $\sim 91 \%, \sim 93 \%$ and $\sim 90 \%$ species of the three categories from NEI (Sharma \& Sharma 2019 b) in particular reveal a closer faunal affinity of the rotifer assemblages of this region with the SE Asian and Australian faunas, and thus assign a distinctive biogeographic identity to NEI Rotifera in contrast with the rest of India. This salient feature is hypothesized as an incursion of various SE Asian and Australian rotifers through 'the Assam gateway' - a unique biogeographic corridor of India (Sharma \& Sharma 2019b). Besides, Indian Rotifera reveals $\sim 41 \%$ species of regional biogeography interest. Of these, Adineta vaga major, A. longicornis, Cephalodella intuta, C. ventripes, Colurella tesselata, Habrotrocha angusticollis, H. angusticollis attenuata, H. lata, H. leitgebii, H. microcephala, Keratella javana, Lecane aeganea, L. clara, L. glypta, L. rhytida, L. stichaea, Lepadella heterodactyla. L. latusinus, L. patella oblonga, Monommata grandis, $M$. maculata, Stephanoceros fimbriatus, Taphrocampa annulosa, Testudinella dendradena, T. tridentata, Trichocerca bidens, T. insignis, T. insulana, T. mus, T. scipio, and T. sulcata are known to-date for their distribution restricted to NEI (Sharma \& Sharma 2019b). Cephalodella panarista, Dicranophorus myriophylli, Floscularia conifera, Hexarthra bulgarica, Itura aurita, Kellicottia longispina, Keratella hiemalis, Lecane elasma, Notholca striata, Notommata aurita, N. copeus, N. tripus, Synchaeta stylata, S. tremula, Testudinella insinuata. T. mucronata, Trichocerca cavia, Trichotria pocillum, and Trochosphaera solstitialis are known exclusively from Jammu \& Kashmir (Sharma \& Sharma 2018a), while Colurella colurus, Euchlanis meneta, Keratella serrulata, K. ticinensis, Mytilina michelangellii, Notholca acuminata, N. labis, and N. squamula indicate validated reports restricted to both the Kashmir Himalayas and NEI (Sharma \& Sharma 2018a, 2019b). In addition, Ascomorpha saltans indica, Cephalodella megalocephala, C. misgurnus, Lophocharis naias, Lepadella kostei, Lecane galeata, L. pawlowskii, L. perplexa, L. schraederi, Notommata pseudocerberus, Proales indirae and Ptygura furcillata are reported only from the state of Gujarat (Wulfert 1966). Our 
remarks thus highlight both the global and regional biogeographic interest of the Indian Rotifera eventhough more interesting species of the two categories are likely to be added pending analyses of extensive collections from hitherto unexplored regions of India and the 'Himalayan, Indo-Burmese and Western Ghats biodiversity hot-spots' in particular.

We consider the current paucity of the Indian endemics to be secondary as: (a) 10 rotifer species i.e. Brachionus srisumonae, Colurella sanoamuangae, Lecane dorysimilis, L. isanensis, L. latissima, L. niwati, L. shieli, L. superaculeata, Lepadella desmeti and Trichocerca siamensis added as new records from India (Sharma \& Sharma 2019b) are originally described as new species from SE Asia; (b) a number of newer taxa observed in our collections from NEI yet await descriptions; (c) the paucity of endemics elsewhere from India is attributed to inadequate sampling particularly of diverse aquatic and semiaquatic environs; and (d) the future collections from the 'Himalayan and Western Ghats biodiversity hot-spots' along with other unexplored / under-explored regions of India are likely to improve the status of the Indian endemics. The reports of the tropical-latitude populations of the Holarctic and Palaearctic species and other coldwater elements from the Himalayas are likely to represent glacial relicts as hypothesized by Segers (1996), while the reports of certain species in our sub-tropical collections are attributed to extension of the Himalayan mountain ranges as hypothesized by Sharma \& Sharma (2014c).

Sharma (1991, 1996, 1998a) focussed attention on state-wise / regional biodiversity disparities. This lacuna still holds valid (Sharma \& Sharma 2017) as highlighted by the fact that amongst 29 states of India, the rotifer assemblages of only 10 states are reasonably well examined, while the regional diversity studies evade attention except for NEI. Referring to NEI, it reveals a total of 303 species (Sharma \& Sharma 2019b), while its seven states namely Assam $>$ Manipur $>$ Tripura, Arunachal Pradesh $>$ Mizoram $\geq$ Meghalaya $>$ Nagaland record 244, 200, 176, 172, 162, 161 and
150 species, respectively. NEI fauna as thus far characterized categorizes it as the most Rotifera biodiverse region of India and also interestingly as one of the most biodiverse in comparison with the countries of south and SE Asia. These salient features are hypothesized to habitat heterogeneity of water bodies located under diverse geoecological regimes of NEI, location of this region under the 'Himalayan' and the 'Indo-Burmese' biodiversity hotspots, 'the Assam gateway' - an interesting biogeographic corridor of India and the sampling intensity (Sharma \& Sharma 2019b), and overall the 'Rotiferologist effect' (Fontaneto et al. 2012). In light of our earlier meta-diversity updates on NEI (Sharma \& Sharma 2005, 2014a, 2019b), we expect more diverse rotifer assemblage of this region pending analyses of the extensive collections from practically unexplored eastern Himalayan state of Sikkim (except for the report of Murray 1906) as well as under-explored eastern Himalayan state of Arunachal Pradesh (Sharma and Sharma 2019a). We also extend our remarks on regional / state wise biodiversity disparities to the states of western India; of these, Jammu \& Kashmir Rotifera records 173 species (Sharma and Sharma 2018a) and offers scope of the future update, and Uttarakhand Rotifera (Sharma 2021) is poorly documented, while Himachal Pradesh and Ladakh yet lack biodiversity surveys on the taxon (BKS, unpublished). Further, we report 177 and 168 species from the states of Tamil Nadu (Sharma \& Sharma 2009) and West Bengal (Sharma 1998b) of east and south India respectively. Our recent studies (BKS unpublished) recording 104 species from Bihar (eastern India); 149, 109 and 64 species from Maharashtra, Madhya Pradesh and Goa, respectively from central India; and 146, 131, 108 and 141 species from Andhra Pradesh. Telengana, Kerala and Karanataka, respectively of south India attempt to augment the regional diversity status but yet suffer from the sampling intensity. Regional disparities of Rotifera biodiversity are influenced by spatial heterogeneity of the speciose monogonont families namely Lecanidae (Sharma \& Sharma 2014b), Brachionidae (Sharma \& Sharma 2014c), Lepadellidae (Sharma \& Sharma 2015b) and Trichocercidae (BKS unpublished). However, we 
focus special attention on paucity of Bdelloidea which are documented to-date vide the limited surveys by Anderson (1889), Murray (1906) and Edmondson \& Hutchinson (1934) and thus deserve specific investigations in the future Rotifera studies from India.

Various Rotifera species examined from India are reported to exhibit morphological variations (Sharma 1983, Sharma and Sharma 2014b, 2014c, 2015a, 2015b, 2018a, 2018c, 2019a). The variations observed in Brachionus angularis, $B$. bidentatus, B. caudatus, B. calyciflorus, $B$. diversicornis, B. falcatus, B. forficula, B. plicatilis, B. quadridentatus, Colurella colurus, C. obtusa, C. uncinata, Epiphanes brachionus, Euchlanis dilatata, Filinia longiseta, F. terminalis, Floscularia ringens, Keratella cochlearis, K. tropica, Lecane bulla, L. closterocerca, L. cornuta, L. curvicornis, L. hamata, L. inermis, L. leontina, L. luna, $L$. lunaris, L. ludwigii, L. monostyla, L. obtusa, L. quadridentata, L. signifera, L. stenroosi, L. ungulata, L. unguitata, Lepadella acuminata, $L$. costatoides, L. ovalis, L. patella, L. rhomboides, L. triptera, Limnias ceratophylli, Plationus patulus, Testudinella amphora, T. emarginula, T. patina, T. tridentata, Trichocerca rattus and T. similis thus await cryptic diversity analyses following some interesting studies (Suatoni et al. 2006; Schröder \& Walsh 2010, Montero-Pau et al. 2011, Mills et al. 2017, Michaloudi et al. 2018) and using 'integrative taxonomic approaches' including 'reverse taxonomy' (Michaloudi et al. 2018). The specific focus on the likely cryptic species complexes is expected to enhance the faunal diversity status of Indian Rotifera.

The rotifer faunal diversity works from India are largely biased to the assemblages from the inland waters of the Indian mainland. In contrast, our exclusive report of 120 species from freshwater rotifers from the south Andaman (Sharma 2017, Sharma et al. 2017) highlights scope of extending such studies to insular freshwaters off other islands of the Andaman and Nicobar group of Islands and the Lakshadweep Islands. The rotifer diversity of the riverine systems is yet poorly known in spite of our highest report of 72 species, belonging to 30 genera and 17 families from an intensively sam- pled $600 \mathrm{~km}$ long stretch of the river Narmada in Madhya Pradesh, Central India (Sharma \& Naik 1996). We also focus attention on inadequately explored rotifer assemblages of the brackish water environs in light of the limited preliminary reports of Rama Rao \& Chandra Mohan (1984), Anitha \& George (2006), Varghese \& Krishnan (2008), George et al. (2011), Varghese (2011), and Cleetus et al. $(2015,2016)$. The biodiversity literature, however, lacks the reports of marine rotifers from India.

Rotifera received attention of majority of 'amateurs' and fewer Indian 'specialists' to-date; the former invariably listed planktonic species from certain ponds, lakes and reservoirs. This trend has hampered holistic analyses of the rotifer assemblages, and the sessile, colonial and bdelloid species in particular, while the littoral-periphytic species are reported in selected studies (Sharma \& Sharma 2017). Further, the Indian literature is loaded with works 'poor illustrations' or 'microphotographs' which fail to enable an objective opinion on actual status of various recorded taxa and thus render them 'unverifiable' (Sharma \& Sharma 2017), while lack of 'voucher specimens' categorize many other reports as 'invalidated and unverifiable'. 'Dubious reports' of species of temperate-centered Keratella, Kellicottia, Nothol$c a$ and Synchaeta, 'unrealistic' and 'fuzzy' reports of Brachionus havanaensis and Keratella valga, and 'incomplete species lists' due to 'overlooking of identification of small species' adversely influence the rotifer biodiversity studies. The 'sloppy and uncritical' descriptions of new taxa, another notable impediment, is highlighted by synonymized 15 new species and subspecies (Sharma \& Sharma 2017), while nine new species are designated as 'species inquirenda' (Segers 2007), Pseudoembata acutipoda Wycliffe \& Michael is treated as 'Genus \& species inquirendus' (Segers 2007, Jersabek \& Leitner 2013) and four species are categorized as 'nomen nudum' (Sharma \& Sharma 2017). Above all, the majority of 'classical taxonomy' based faunal works highlight need to shift to 'integrative taxonomic approaches' for the effective biodiversity updates on Indian Rotifera. 
Analyses of the rotifer diversity from diverse aquatic environs of India remained neglected until certain noteworthy inputs from the floodplains lakes (beels and pats) of NEI (Sharma \& Sharma 2001, 2008, 2014a, 2014d, 2019b, Sharma 2005, 2009a, 2009b, 2014, unpublished) resulting in the reports of 244 species belonging to 46 genera and 21 families from the beels of Assam, and 210 species belonging to 48 genera and 23 families from pats of Manipur. We, hypothesize these biodiverse Rotifera assemblages to habitat diversity and environmental heterogeneity of the floodplain lakes of NEI located in the 'IndoBurmese biodiversity hot-spot'. Further, Assam beels record more speciose rotifers and those of Manipur pats are marginally diverse than the reports of 207 (Segers et al. 1993) and 218 (Bonecker et al. 1998) species from the floodplains of Africa and South America, respectively. Our results endorse hypothesis of Segers et al. (1993) on the floodplain lakes as the globally important rotifer habitats. Besides, our collections affirm the fairly speciose Rotifera of the Kashmir valley (140 species: Sharma \& Sharma 2018a) and West Bengal (152 species: BKS, unpublished) floodplains, while the floodplain lakes elsewhere from India are yet to adequately surveyed for Rotifera biodiversity.

Loktak Lake (Sharma \& Sharma 2018c, 2019a, BKS unpublished) and Deepor Beel (Sharma \& Sharma 2011, 2012, 2013, 2015c, BKS unpublished), only two intensively sampled Ramsar sites of India record 203 and 191 species respectively, and are thus categorized as the globally megadiverse Rotifera ecosystems. These important floodplain lakes of NEI depict biodiverse Rotifera than the Rio Pilcomayo National Park (114 species, Jose de Paggi 2001) and Thale-Noi Lake (106 species, Segers \& Pholpunthin 1997) the Ramsar sites of Argentina and Thailand, respectively as well as the well sampled Upper Paraná floodplain of Brazil (184 species; Bonecker et al. 2005). Our reports from Loktak Lake and Deepor Beel assume distinct national importance in contrast to the poor state-of-art status of the rotifer biodiversity of rest of 40 Ramsar sites of India (Chandra et al. 2021). The comparisons highlight importance of extension of studies on Rotifera assemblages from other Ramsar sites of this country vis-à-vis potential to augment the biodiversity status of Indian fauna. Interestingly, our reports of 85 species each in December 2016 and January 2017 samples from Deepor beel and 86 and 89 species in November and December 2017 collections from Loktak Lake (Sharma \& Sharma 2019b), and 84 and 81 species (during May and June, 2017) from a floodplain lake (beel) of upper Assam highlight speciose rotifer constellations per sample. We designate the speciose constellations of $80+$ species per sample as the 'Rotifera paradox' as these depict examples of intriguing possibility of the co-existence of a number of species due to high amount of niche overlaps as hypothesized (MacArthur 1965).

The small water bodies (ponds and wetlands) are considered as keystone systems for analyses of biodiversity (Vad et al. 2017; Oertli 2018). Our collections from small floodplain wetlands (dobas or dubies) of the Brahmaputra and the Barak river basins of Assam state of NEI report 167 and 156 species, respectively and thus highlight the speciose rotifers. Besides, our reports of 165,150 , 162 and 161 species highlight species-rich rotifer assemblages of small lentic biotopes (wetlands) predominant in the hill states of Arunachal Pradesh, Nagaland, Mizoram and Meghalaya states of NEI, respectively (Sharma \& Sharma 2019b), while 163 and 168 species known from small wetlands of Tripura and Manipur states of NEI, respectively also endorse our results. Hill et al. (2017) hypothesized the importance of small urban wetlands for maintaining regional biodiversity in highly modified urban environments. This hypothesis is affirmed by 90 species reported from a small urban wetland of Meghalaya state (Sharma \& Sharma 2021). In general, the biodiversity importance of dobas or dubies, and other small and urban wetlands of NEI are attributed to habitat heterogeneity of these unstructured environs and the sampling intensity. Our collections from dobas or dubies of the Majuli River Island and those of upper Assam highlight 'Rotifera paradox' with the speciose assemblages of up to 50 species per sample (Sharma \& Sharma 
2019b, 2019c, 2019d). Besides, our report of 46 species per sample from small urban wetland of Meghalaya (Sharma \& Sharma 2021) is categorized as 'Rotifera paradox' analogous to the reports (Sharma \& Sharma, 2019b, 2019c, 2019b) from unstructured dobas or dubies.

To sum up, the rich and diverse Indian Rotifera highlights its biodiversity interest and notable fractions of species of the global and regional distribution interest impart biogeography importance. The rotifer fauna of India as thus far characterized reflects the littoral-periphytic nature and broadly tropical character though certain cold-water species are observed from the subHimalayan and Himalayan latitudes. We highlight the disparity of the state wise / regional biodiversity studies, inadequate analyses of bdelloid rotifers, and spatial heterogeneity of the richness. NEI is notable for the richest regional Rotifera diversity; Loktak Lake and Deepor beel deserve global attention as the rotifer megadiverse environs; and the floodplain lakes (beels and pats), the small floodplain wetlands (dobas or dubies) and small lentic environs depict the speciose assemblages with interesting instances of 'Rotifera paradox'. The biodiversity status of Indian Rotifera yet needs to be augmented based on the intensive sampling of unexplored or underexplored states / regions / ecosystems, the 'Himalayan and Western Ghats biodiversity hotspots', and various 'Ramsar' sites of India. Analyses of cryptic diversity as well as adequate focus on the sessile, colonial, benthic and littoralperiphytic assemblages, and identification of smaller species deserve attention. As per conservative estimate, we expect the Indian Rotifera tally of $550+$ species pending attention to various lacunae.

Acknowledgements - This contribution is dedicated to advice and guidance received from an eminent Rotiferologist, the late Dr. Walter Koste, Germany. The senior author thanks the Head, Department of Zoology, NEHU, Shillong for the laboratory facilities, and collectively to all those who helped in the field studies during the last three decades. We extend our sincerest thanks to Prof. Elizabeth Walsh, University of Texas at El Paso, USA for critical scrutiny of our manuscript. We thank our anonymous reviewers for useful comments and suggestions. The authors have no conflict of interests.

\section{REFERENCES}

ANDERSON, H.H. (1889): Notes on Indian Rotifera. Journal of the Asiatic Society of Bengal, 58: 345358.

ANITHA, P.S. \& GEORGE, R.M. (2006): The taxonomy of Brachionus plicatilis species complex (Rotifera, Monogononta) from the Southern Kerala (India) with a note on their reproductive preferences. Journal of the Marine Biological Association India, 48(1): 6-13.

ARORA, J. \& MEHRA, N.K. (2003): Species diversity of planktonic and epiphytic rotifers in the backwaters of the Delhi segment of the Yamuna River, with remarks on new records from India. Zoological Studies, 42(2): 239-247.

BANIK, S. (2000): Two new species of genus Collotheca Harring 1913 (Rotifera, Monogononta) from freshwaters of Tripura, India. Journal of the Bombay Natural History Society, 99(2): 262-266

Bonecker, C.C., Costa, C.L.D., Velho, L.F.M. \& LANSAC-TÔHA, F.A. (2005): Diversity and abundance of the planktonic rotifers in different environments of the Upper Paraná River floodplain (Paraná State - Mato Grosso do Sul State, Brazil). Hydrobiologia, 546: 405-414. doi: 10.1007/s10750$\underline{005-4283-2}$

BONECKER, C.C., LANSAC-TÔHA, F.A. \& ROSSA, F.C. (1998): Planktonic and non-planktonic rotifers in two environments of the upper Parana river floodplain, state of Mato Grosso do Sul, Brazil. Brazilian Archives of Biology \& Technology, 41: 447-456. doi: $\underline{10.1590 / \mathrm{S} 1516-89131998000400009}$

ChANDRA, K., BHARTI, D., KUMAR, S., RAGHUNATHAN, C., GUPTA, D., ALFRED, J.R.B. \& Chowdhury, B.R. (2021): Faunal Diversity in Ramsar Wetlands of India. Jointly published by Zoological Survey of India, and Wetland division, Ministry of Environment, Forest and Climate Change, Government of India, 292 pp.

Cleetus, R.I, Asha, C, Suson, P.S. \& Bijoy NanDAN, S. (2015): Species diversity and community assemblage of planktonic rotifers from Vembanad estuary - Kerala, India. International Journal of Oceanography and Marine Ecological System, 4(1): 1-15.

Cleetus, R.I, Asha, C.V., Suson, P.S. \& Bijoy NANDAN, S. (2016): Mesozooplankton abundance and community structure in Vembanad-Kol wetland 
ecosystem, Kerala, India. Indian Journal of GeoMarine Sciences, 45(4): 533-545.

DE RIDDER, M. (1981): Some considerations on the geographical distribution of Rotifera. Hydrobiologia, 85: 209-235. doi: 10.1007/BF00017611

DHANAPATHI, M.V.S.S.S. (1974): A new brachionid rotifer Platyias quadricornis andhraensis subsp. nov. from India. Current Science, 43: 358.

DHANAPATHI, M.V.S.S.S. (1975): Rotifers from Andhra Pradesh, India. Zoological Journal of the Linnaean Society, 57 (1): 85-94. doi: $\underline{10.1111 / \mathrm{j} .1096-3642.1975 . t b 01892 . \mathrm{x}}$

DHANAPATHI, M.V.S.S.S. (1976): A new lecanid rotifer from India. Hydrobiologia, 50: 191-192. doi: 10.1007/BF00019823

DHANAPATHI, M.V.S.S.S. (1978): New species of rotifer from India belonging to the family Brachionidae. Zoological Journal of the Linnaean Society, 63: 305-308. doi: 10.1111/j.1096-3642.1978.tb01041.x

DHANAPATHI, M.V.S.S.S. (2000): Taxonomic notes on the rotifers from India (from1889-2000). Indian Association of Aquatic Biology Publication, 10: 1178.

DUMONT, H.J. (1983): Biogeography of rotifers. Hydrobiologia, 104: 19-30. doi: $10.1007 / \mathrm{BF} 00045948$

Dussart, B.H., FERNANDO, C.H., MATSUMURATUNDISI, J. \& SHIEL, R.J. (1984): A review of systematics, distribution and ecology of tropical freshwater zooplankton. Hydrobiologia, 113: 7791. doi: $10.1007 / \mathrm{BF} 00026594$

Edmondson, W.T. \& HutCHInson, G.E. (1934): Report on Rotatoria. Article IX. Yale North India Expedition. Memories of the Connecticut Academy of Arts and Sciences, 10: 153-186.

FERNANDO, C.H. (1980): The freshwater zooplankton of Sri Lanka, with a discussion of tropical freshwater zooplankton composition. Internationale Revue des Hydrobiologie, 65: 411-426. doi: 10.1002/iroh.19800650105

FontANETO, D. \& RiCCI, C. (2004): Rotifera: Bdelloidea. In. Yule, C.M. \& SEN, Y.H. (Eds.) Freshwater Invertebrates of the Malaysian Region. Academy of Sciences Malaysia, p. 121-126.

Fontaneto, D., Márcia Barbosa, A, Segers, H. \& PAUTASSO, M. (2012): The 'rotiferologist' effect and the other global correlates of species richness in rotifers. Ecography, 35: 174-182. doi: $10.1111 /$ j. $1600-0587.2011 .06850 . x$
George, G., Sreeraj, C.R. \& DAM Roy, S. (2011): Brachionid rotifer diversity in Andaman waters. Indian Journal of Geo-Marine Sciences, 40(3): 454-459.

GREEN J. (1972): Latitudinal variation in associations of planktonic Rotifera. Journal Zoological Society London, 167: 31-39. doi: $10.1111 / \mathrm{j} .1469-7998.1972 . t b 01721 . x$

GREEN, J. (2003): Associations of planktonic and periphytic rotifers in a tropical swamp, the Okavango Delta, Southern Africa. Hydrobiologia, 490: 197209. doi: $10.1023 / \mathrm{A}: 1023475217987$

Hill, M.J., Biggs, J., ThORnhill, I., Briers, R.A., Gledhill, D.G., White, J.C., WoOD, P.J. \& HASSALL, C. (2017): Urban ponds as an aquatic biodiversity resource in modified landscapes. Global Change Biology, 23(3): 986-999. doi: $\underline{10.1111 / \mathrm{gcb} .13401}$

JERSABEK, C.D \& LEITNER, M.F. (2013): The Rotifer World Catalog. World Wide Web electronic publication. http://www.rotifera.hausdernatur.at/, accessed (03.12.2020)

José De PAGGI, S. (2001): Diversity of Rotifera (Monogononta) in wetlands of Rio Pilcomayo national park, Ramsar site (Formosa, Argentina). Hydrobiologia, 462: 25-34. doi: 10.1023/A:1013157914860

Koste, W. (1978): Rotatoria. Die Rädertiere Mitteleuropas, begründet von Max Voigt. Überordnung Monogononta. Gebrüder Borntraeger, Berlin, Stuttgart. I. 673 pp., II. Tafelband. 234 pp.

Koste, W. \& SHIEL, R.J. (1987): Rotifera from Australian inland waters. II. Epiphanidae and Brachionidae (Rotifera, Monogononta). Invertebrate taxonomy, 7: 949-1021. doi: 10.1071/IT9870949

Koste, W. \& SHIEL, R.J. (1989): Rotifera from Australian inland waters. IV. Colurellidae (Rotifera, Monogononta). Transactions of the Royal Society of South Australia, 113: 119-143.

Koste, W. \& ShIEL, R.J. (1990): Rotifera from Australian inland waters. V. Lecanidae (Rotifera, Monogononta). Transactions of the Royal Society of South Australia, 114: 1-36.

MACARTHUR, R.H. (1965): Patterns of species diversity. Biological Review, 40: 510-533. doi: 10.1111/j.1469-185x.1965.tb00815.x

Michaloudi, E., Papakostas, S., Stamou, G., NeDĚLA, V., TIHLAŘíKOVÁ, A.E., ZHANG, W. \& DECLERCK, A.J. (2018): Reverse taxonomy applied to 
the Brachionus calyciflorus cryptic species complex: Morphometric analysis confirms species delimitations revealed by molecular phylogenetic analysis and allows the (re) description of four species. PLoS ONE, 13(9): e0203168.

doi: $\underline{10.1371 / \text { journal.pone. } 0203168}$

Mills, S., AlcÁNTARA-RodríGuez, J.A., Ciros-PÉREZ, J., Gómez, A., Hagiwara, A., Galindo, K.H., JersabeK, C.D., MaleKZaDEH-VIAYEH LeASI, R.F., LEE, J.S., MARK WELCh, D.B., PAPAKostas, S., Riss S., SEgers, H., SERra, M., Shiel, R., SMOlaK, R., SNell, T.W., Stelzer, C.P., TANG, C.Q., Wallace, R.L., Fontaneto, D. \& WALSH E.J. (2017): Fifteen species in one: deciphering the Brachionus plicatilis species complex (Rotifera, Monogononta) through DNA taxonomy. Hydrobiologia, 96: 39-58. doi: 10.1007/s10750-016$\underline{2725-7}$

Montero-Pau, J., Ramos-Rodríguez, E., Serra, M. \& GómEZ, A. (2011): Long-term coexistence of rotifer cryptic species. PLOS ONE, 6: e21530. doi: 10.1371/journal.pone.0021530

Murray, J. (1906): Some Rotifera of the Sikkim Himalaya. Journal of the Royal Microscopical Society London, 2(9): 637-644. doi: 10.1111/j.13652818.1906.tb00619.x

NAYAR. C.K.G. (1968): Rotifers fauna of Rajasthan, India. Hydrobiologia, 31(2): 168-185. doi: $\underline{10.1007 / \mathrm{BF} 00206050}$

OERTLI B. (2018): Freshwater biodiversity conservation: The role of artificial ponds in the 21 st century. Aquatic Conservation: Marine and Freshwater Ecosystems, 28(2): 264-269. doi: 10.1002/aqc.2902

PEJLER B. (1977): On the global distribution of the family Brachionidae (Rotatoria). Archiv für Hydrobiology, Supplement, 53: 255-306.

RAMA RAO. K. \& Chandra Mohan, P. (1984): Brackish water Rotifera from Visakhapatnam harbor. Indian Journal of Marine Sciences, 13: 92-93.

SA-Ardrit, P., Pholpunthin, P. \& Segers, H. (2013): A checklist of the freshwater rotifer fauna of Thailand (Rotifera, Monogononta, Bdelloidea). Journal of Limnology, 72(2): 361-375. doi: 10.4081/ jlimnol.2013.s2.e18

SCHRÖDER, T. \& WALSH E.J. (2010): Genetic differentiation, behavioural reproductive isolation and mixis cues in three sibling species of monogonont rotifers. Freshwater Biology, 55: 2570-2584. doi: 10.1111/j.1365-2427.2010.02487.x
SEGERS, H. (1995): Rotifera 2: Lecanidae. In. DUMONT, H.J. \& NoGRADY, T. (Eds.) Guides to identification of the Microinvertebrates .of the Continental waters of the world, 6. SPB Academic Publishing bv. Amsterdam, the Netherlands, 226 pp.

SEGERS, H. (1996): The biogeography of littoral Lecane Rotifera. Hydrobiologia, 323: 169-197. doi: $\underline{10.1007 / \mathrm{BF} 00007845}$

SEGERS, H. (2001): Zoogeography of the Southeast Asian Rotifera. Hydrobiologia, 446/ 447: 233-246. doi: $10.1023 / \mathrm{A}: 1017524011783$

SEGERS, H. (2002): The nomenclature of the Rotifera, annotated checklist of valid family- and genusgroup names. Journal of Natural History, 36: 621640. doi: $10.1080 / 002229302317339707$

SEgERS, H. (2004): Rotifera: Monogononta. In. Yule CM \& SEN YH. (Eds.) Freshwater Invertebrates of the Malaysian Region. Academy of Sciences Malaysia, p. 106-119.

SEGERS, H. (2007): Annotated checklist of the rotifers (Phylum Rotifera), with notes on nomenclature, taxonomy and distribution. Zootaxa, 1564: 1-104. doi: $\underline{10.11646 / \text { zootaxa.1564.1.1 }}$

SEGERS, H. (2008): Global diversity of rotifers (Rotifera) in freshwater. Hydrobiologia, 595: 49-59. doi: $10.1007 / \mathrm{s} 10750-007-9003-7$

Segers, H, Nwadiaro, C.S. \& Dumont, H.J. (1993): Rotifera of some lakes in the floodplain of the river Niger (Imo State, Nigeria). II. Faunal composition and diversity. Hydrobiologia, 250: 63-71. doi: $\underline{10.1007 / \mathrm{BF} 00007495}$

Segers, H. \& Pholpunthin, P. (1997): New and rare Rotifera from Thale Noi Lake, Pattalang Province, Thailand, with a note on the taxonomy of Cephalodella (Notommatidae). Annals de Limnologie, 33: 13-21. doi: 10.1051/limn/1997001

SHARMA, B.K. (1983): The Indian species of the genus Brachionus (Eurotatoria: Monogononta: Brachionidae). Hydrobiologia, 104: 31-39. doi: $10.1007 / \mathrm{BF} 00045949$

SHARMA, B.K. (1987a): Indian Brachionidae (Eurotatoria: Monogononta) and their distribution. Hydrobiologia, 144: 269-275. doi: 10.1007/BF00005561

SHARMA, B.K. (1987b): On the distribution of lecanid rotifers (Rotifera: Monogononta: Lecanidae) in North-Eastern India. Revue d'hydrobiologie tropicale, 20: 101-105. 
SHARMA, B.K. (1991): Rotifera. In. Animal Resources of India: Protozoa to Mammalia: State of the Art. Zoological Survey of India, Calcutta, p. 69-88.

SHARMA, B.K. (1996): Biodiversity of Freshwater Rotifera in India - a status report. Proceeding of the Zoological Society Calcutta, 49 (2): 73-85.

Sharma, B.K. (1998a): Rotifera. In. Alfred, J.R.B., DAS, A.K. \& SANYAL, A.K. (Eds.). Faunal Diversity of India. ENVIS Centre, Zoological Survey of India, Calcutta, p. 57-70.

SHARMA, B.K. (1998b): Freshwater Rotifers (Rotifera: Eurotatoria). Fauna of West Bengal, State Fauna Series, Zoological Survey of India, Calcutta, 3(11): 341-461.

SHARMA, B.K. (2004): Rare and interesting monogonont rotifers (Rotifera: Eurotatoria) from NorthEastern India. Zoosystematics \& Evolution, 80(1): 33-40. doi: 10.1002/mmnz.20040800103

SHARMA, B.K. (2005): Rotifer communities of floodplain lakes of the Brahmaputra basin of lower Assam (N. E. India): biodiversity, distribution and ecology. Hydrobiologia, 533: 209-221. doi: 10.1007/s10750$\underline{004-2489-3}$

SHARMA, B.K. (2009a): Rotifer communities of floodplain lakes of Manipur (North-East India), biodiversity, distribution and ecology. Journal of the Bombay Natural History Society, 106(1): 45-56.

SHARMA, B.K. (2009b): Diversity of Rotifers (Rotifera: Eurotatoria) of Loktak lake, North-Eastern India. Tropical Ecology, 50(2): 277-285

SHARMA, B.K. (2014): Rotifers (Rotifera: Eurotatoria) from wetlands of Majuli - the largest river island, the Brahmaputra river basin of upper Assam, northeast India. Check List, 10(2): 292-298. doi: $\underline{10.15560 / 10.2 .292}$

SHARMA, B.K. (2017): First report of freshwater Rotifers (Rotifera: Eurotatoria) from south Andaman, India: composition and interesting elements. Journal of the Asia-Pacific Biodiversity, 10(2): 261-266. doi: 10.1016/j.japb.2017.01.003

SHARMA, B.K., HAOKIP, T.P. \& SHARMA, S. (2016): Loktak Lake, Manipur, northeast India: a Ramsar site with rich rotifer (Rotifera: Eurotatoria) diversity and its meta-analysis. International Journal of Aquatic Biology, 4(2): 69-79. doi: $10.22034 / \mathrm{ijab} . v 4 \mathrm{i} 2.158$
Sharma, B.K., KHAN, S.I. \& SHARMA, S. (2018): Biodiverse rotifer assemblage (Rotifera: Eurotatoria) of floodplain lakes of the Brahmaputra basin of lower Assam, northeast India: composition and ecosystem diversity. Journal of Oceanology and Limnology, 36(2): 362-375. doi: 10.1007/s00343-017-6251-x

SHARMA, B.K., KENSIBO, \& SHARMA, S. (2017): Biodiversity of rotifers (Rotifera: Eurotatoria) of Nagaland, northeast India; richness, composition and ecosystem diversity. International Journal of Fisheries \& Aquatic Studies, 5(5): 180-187.

Sharma, B.K. \& Michael, R.G. (1980): Synopsis of taxonomic studies on Indian Rotatoria. Hydrobiologia, 73: 229-236. doi: 10.1007/BF02530262

SHARMA, B.K. \& NAIK, L.P. (1996): Results on planktonic Rotifers in the Narmada river (Madhya Pradesh, Central India). In. ScHIMER, F. \& BOLAND, K.T. (Eds.) Perspectives in Tropical Limnology, SPB Academic Publishing bv. Amsterdam, the Netherlands, p. 189-198.

SHARMA, B.K., NOROH, N. \& Sharma, S. (2017): Rotifers (Rotifera: Eurotatoria) from floodplain lakes of the Dibru Saikhowa Biosphere Reserve, upper Assam, northeast India: ecosystem diversity and biogeography. International Journal of Aquatic Biology, 5(2): 79-94. doi: 10.22034/ijab.v5i2.281

SHARMA, B.K., RAGHUNATHAN, C. \& SHARMA, S. (2017): Rich freshwater Rotifer (Rotifera: Eurotatoria) fauna of small lentic ecosystems of south Andaman, India. Opuscula Zoologica Budapest, 48, (2): 185-192. doi: 10.18348/opzool.2017.2.185

SHARMA, B.K. \& SHARMA, S. (1987): On species of the genus Lepadella (Eurotatoria: Monogononta: Colurellidae) from North-Eastern India, with remarks on Indian taxa. Hydrobiologia, 147:15-22. doi: $\underline{\text { 10.1007/BF00025721 }}$

SHARMA, B.K. \& SHARMA, S. (1999): Freshwater rotifers (Rotifera, Eurotatoria). Fauna of Meghalaya, State Fauna Series, Zoological Survey of India, Calcutta, 4(9): 11-161.

ShARMA, B.K. \& Sharma, S. (2000): Freshwater rotifers (Rotifera, Eurotatoria). Fauna of Tripura, State Fauna Series, Zoological Survey of India, Calcutta, 7(4): 163-224.

SHARMA, B.K. \& SHARMA, S. (2001): Biodiversity of Rotifera in some tropical floodplain lakes of the 
Brahmaputra river basin, Assam (N. E. India). Hydrobiologia, 446 / 447: 305-313. doi: 10.1023/A: 1017517822377

SHARMA, B.K. \& SHARMA, S. (2005): Biodiversity of freshwater rotifers (Rotifera, Eurotatoria) from North-Eastern India. Zoosystematics \& Evolution, 81: 81-88. doi: 10.1002/mmnz.200310002

Sharma, B.K. \& SHARMA, S. (2009): Biodiversity and distribution of freshwater rotifers (Rotifera, Eurotatoria) of Tamil Nadu. Records of the Zoological Survey of India, 109(3): 41-60.

SHARMA, B.K. \& SHARMA, S. (2011): Deepor Beel revisited: new records of rotifers (Rotifera, Eurotatoria) with remarks on interesting species. Journal of Threatened Taxa, 3(1): 1437-1444. doi: $\underline{10.11609 / J o T T .02482 .1437-44}$

SHARMA, B.K. \& SHARMA, S. (2012): Deepor Beel - a Ramsar site of India, an interesting hot-spot with its rich Rotifera biodiversity. Acta Zoologica Academiae Scientiarum Hungaricae, 58(2): 105-120.

SHARMA, B.K. \& SHARMA, S. (2014a): Northeast India - An important region with a rich biodiversity of Rotifera. In. SHARMA, B.K., DUMONT, H.J. \& WALLACE, R.L. (Eds.) Rotifera XIII: Rotifer Biology - A structural and functional Approach. International Review of Hydrobiology, 99(1-2): 20-37. doi: $\underline{\text { 10.1002/iroh.201301701 }}$

ShARMA, B.K. \& SHARMA, S. (2014b): Indian Lecanidae (Rotifera: Eurotatoria: Monogononta) and its distribution. In. SHARMA, B.K., DUMONT, H.J. \& WALLACE, R.L. (Eds.), Rotifera XIII: Rotifer Biology - a Structural and Functional Approach. International Review of Hydrobiology, 99(1-2): 38-47. doi: 10.1002/iroh.201301702

SHARMA, B.K. \& SHARMA, S. (2014c): The diversity of Indian Brachionidae (Rotifera: Eurotatoria: Monogononta) and their distribution. Opuscula Zoologica Budapest, 45(2): 165-180.

SHARMA, B.K. \& SHARMA, S. (2014d). Floodplains of the Brahmaputra river basin - globally interesting ecotones with rich Rotifer (Rotifera: Eurotatoria) biodiversity. In. SINHA, R.K. \& AHMED, B. (Eds.), Rivers for Life - Proceedings of the International Symposium on River Biodiversity: Ganges-Brahmaputra-Meghna River System, Ecosystems for Life, A Bangladesh - India Initiative, IUCN, International Union for Conservation of Nature, $\mathrm{p}$. 258-270.
SHARMA, B.K. \& SHARMA, S. (2015a): Biodiversity of freshwater rotifers (Rotifera: Eurotatoria) of Mizoram, Northeast India: composition, new records and interesting features. International Journal of Aquatic Biology, 3(5): 301-313. doi: 10.22034/ijab.v3i5.109

SHARMA, B.K. \& SHARMA, S. (2015b): The diversity and distribution of Lepadellidae (Rotifera: Eurotatoria: Monogononta) of India. International Review of Hydrobiology, 100(1): 34-42. doi: 10.1002/iroh.201401739

SHARMA, B.K. \& SHARMA, S. (2015c): New records of rotifers (Rotifera: Eurotatoria) from Deepor beel-a Ramsar site of India with an update on its Rotifera diversity. Journal of Threatened Taxa 7(3): 70117016. doi: 10.11609/JoTT.o4044.7011-6

SHARMA, B.K. \& SHARMA, S. (2017): Rotifera: Eurotatoria (Rotifers). In. CHANDRA, K., GOPI, K.C., RAO, D.V., VAlarmathi, K. \& AlFRED, J.R.B. (Eds.) Current status of freshwater faunal diversity in India, Zoological Survey of India, Kolkata, Chapter 7, p. 93-113.

SHARMA, B.K. \& SHARMA, S. (2018a): The rotifers (Rotifera: Eurotatoria) from the Kashmir Himalayan floodplains and Rotifera biodiversity of Jammu \& Kashmir, north India. International Journal of Aquatic Biology, 6(4): 208-220. doi: 10.22034/ijab.v6i4.507

SHARMA, B.K. \& SHARMA, S. (2018b): The Indian species of Testudinella (Rotifera: Flosculariacea: Testudinellidae) and their distribution. International Journal of Aquatic Biology, 6(1): 15-20. doi: 10.22034/ijab.v6i1.401

SHARMA, B.K. \& SHARMA, S. (2018c): Loktak Lake, Manipur revisited: A Ramsar site as the rotifer (Rotifera: Eurotatoria) biodiversity hot-spot of the Indian sub-region. Bonn zoological Bulletin, 67(1): 5-13.

Sharma, B.K. \& Sharma, S. (2019a): The biodiverse rotifer assemblages (Rotifera: Eurotatoria) of Arunachal Pradesh, the eastern Himalayas: alpha diversity, distribution and interesting features. Bonn zoological Bulletin, 68(1):1-12. doi: 10.20363/bzb-2019.68.1.01

SHARMA, B.K. \& SHARMA, S. (2019b): The biodiverse rotifers (Rotifera: Eurotatoria) of Northeast India: faunal heterogeneity, biogeography, ecosystem diversity and interesting species assemblages. Bonn zoological Bulletin, 68(1):147-162. doi: $10.20363 /$ BZB-2019.68.1.147 
SHARMA, B.K. \& SHARMA, S. (2019c): The biodiverse rotifers (Rotifera: Eurotatoria) of the floodplain wetlands of Barak valley of Assam state, northeast India. Opuscula Zoologica Budapest, 50(1): 3-15. doi: 10.18348/opzool.2019.1.3

SHARMA, B.K. \& SHARMA, S. (2019d): The biodiverse rotifers (Rotifera: Eurotatoria) from small wetlands of the Brahmaputra river floodplains of lower and upper Assam, northeast India. Journal of Limnology \& Freshwater Fisheries Research, 5(3): 187-196. doi: 10.17216/LimnoFish-15981

SHARMA, B.K. \& SHARMA, S. (2021): Zooplankton diversity of a sub-tropical small urban wetland of Meghalaya, northeast India with remarks on spatial variations. Journal of Limnology \& Freshwater Fisheries Research, 7(1): 24-39. doi: 10.17216/LimnoFish-754060

SHARMA, R.C. (2021): Habitat ecology and diversity of freshwater zooplankton of Uttarakhand Himalaya, India. Biodiversity international journal, 4(5): 188196. doi: $10.15406 /$ bij.2020.04.00184

SHARMA, S. \& SHARMA, B.K. (2008): Zooplankton diversity in floodplain lakes of Assam. Records of the Zoological Survey of India, Occasional Paper No. 290: 1-307.

Sharma, S. \& SHARMA, B.K. (2013): Faunal diversity of aquatic invertebrates of Deepor Beel (a Ramsar site), Assam, northeast India. Wetland Ecosystem Series, Zoological Survey of India, Kolkata, 17: 1226.

ShIEL, R,J. \& Koste, W. (1992): Rotifera from Australian inland waters VIII. Trichocercidae (Monogononta). Transactions of the Royal Society of South Australia, 116: 1-27.
Sor, R., Meas, S., Wong, K.Y., Min, M. \& SEgers, H. (2015): Diversity of Monogononta rotifer species among standing water bodies in northern Cambodia. Journal of Limnology, 74(1): 192-204. doi: $\underline{10.4081 / j \text { limnol.2014.995 }}$

Suatoni, E., Vicario, S., Rice, S., Snell, T. \& CaCCONE, A. (2006): An analysis of species boundaries and biogeographic patterns in a cryptic species complex: The rotifer - Brachionus plicatilis. Molecular Phylogenetics and Evolution, 41: 86-98. doi: 10.1016/j.ympev.2006.04.025

Trinh, M.D., Vo, M.V., TraW, A.N.Q., LE, H.T.N. \& TRAN, S.N. (2019): Species diversity of rotifers (Rotifera: Eurotatoria) of Phu Ninh Lake with five new records from Vietnam. International Journal of Aquatic Biology, 7(1): 38-44. doi: 10.22034/ijab.v7i1.581

VAD, Cs.F., PÉNTEK, A., COZMA, N.J., FÖLDI, A., TÓTH, A., Tóth, B., BÖDE, N.A., MóRA, A., PTACNIK, R., ÁCs, E., ZsugA, K. \& HorVÁth, Zs. (2017): Wartime scars or reservoirs of biodiversity? The value of bomb crater ponds in aquatic conservation. Biological Conservation, 209: 253-262. doi: $\underline{10.1016 / j . b i o c o n} .2017 .02 .025$

VARGHESE, M. (2011): Distribution of Brachionus species (Phylum Rotifera) in Cochin backwaters. Kerala, India. Journal of the Marine Biological Association of India, 53 (1):130-134.

VARGHESE, M. \& KRISHNAN, L. (2008): A preliminary study on rotifer fauna of Cochin backwaters, Kerala. Journal of the Marine Biological Association of India, 50 (1): 119-121

WulfERT, K. (1966): Rotatorien aus dem Stausse Azwa und der Trinkwasser Aufbereitung der Stadt Baroda (Indien). Limnologica, 4:53-93. 